\title{
Detailed spectroscopic analysis of the Trapezium cluster stars inside the Orion nebula
}

\section{Rotational velocities, stellar parameters, and oxygen abundances ${ }^{\star}, \star \star$}

\author{
S. Simón-Díaz ${ }^{1,2}$, A. Herrero ${ }^{1,3}$, C. Esteban ${ }^{1,3}$, and F. Najarro ${ }^{4}$ \\ 1 Instituto de Astrofísica de Canarias, 38200 La Laguna, Tenerife, Spain \\ e-mail: ssimon@iac.es \\ 2 Isaac Newton Group of Telescopes, Apartado de Correos 321, 38700 Santa Cruz de la Palma, Tenerife, Spain \\ 3 Departamento de Astrofísica, Universidad de La Laguna, Avda. Astrofísico Francisco Sánchez, s/n, 38071 La Laguna, Spain \\ ${ }^{4}$ Instituto de Estructura de la Materia, CSIC, C/ Serrano 121, 28006 Madrid, Spain
}

Received 15 March 2005 / Accepted 1 October 2005

\section{ABSTRACT}

\begin{abstract}
We present the results of a spectroscopic analysis of the Trapezium cluster stars inside the Orion nebula. The rotational velocities were obtained using the Fourier analysis method, and we found agreement with values derived by the usual method based on linewidth measurements. The rotational velocity derived for $\theta^{1}$ Ori $\mathrm{C}$ by this method is consistent with the variability of some of its spectral features that have a period of 15.42 days. By means of the fit of $\mathrm{H}, \mathrm{He}$, and He II observed profiles with FASTWIND synthetic profiles, stellar parameters and wind characteristics were derived. This methodology let us estimate the errors associated with these parameters, and we found that macroturbulence effects have to be included for a good fit to the He I-II lines in the spectrum of $\theta^{1}$ Ori C.

By means of a very accurate study, oxygen abundances were derived for the three B0.5V stars $\theta^{1}$ Ori A, D, and $\theta^{2}$ Ori B. Final abundances are consistent with the nebular gas-phase results presented in Esteban et al. (2004) and are lower than those given by Cunha \& Lambert (1994). Our results suggest a lower dust depletion factor of oxygen than previous estimations for the Orion nebula.
\end{abstract}

Key words. stars: abundances - stars: early-type - stars: fundamental parameters - ISM: abundances - ISM: H II regions ISM: individual objects: Orion nebula

\section{Introduction}

New developments in massive-star model-atmosphere codes have led to interesting new possibilities for stellar spectroscopic studies. Improvements in computational methods, as well as an increase in the efficiency of computers, have made it possible to model the atmospheres of hot luminous stars, taking into account not only strong NLTE effects and hundreds of thousands of metallic lines that produce the so-called lineblanketing (Hubeny \& Lanz 1995), but also winds with expanding spherical geometries (Santolaya-Rey et al. 1997; Hillier \& Miller 1998; Pauldrach et al. 2001; Puls et al. 2005).

The new improvements included in these latest generation models call for a review of previous results. For example,

$\star$ The INT is operated on the island of La Palma by the RGO in the Spanish Observatory of El Roque de los Muchachos of the Instituto de Astrofísica de Canarias.

$\star \star$ Figures 4 to 10 are only available in electronic form at http://www. edpsciences.org the papers by Herrero et al. (2002), Crowther et al. (2002), and Martins et al. (2002) show that the SpT $-T_{\text {eff }}$ calibrations used previously (Vacca et al. 1996) needed to be revised to lower effective temperatures for a given spectral type. Recent analyses by Repolust et al. (2004) and Martins et al. (2005) in the Milky Way, and by Massey et al. $(2004,2005)$ at SMC and LMC metallicities, reinforce this conclusion and together imply a need to revisit the ionizing flux distribution that is used for the study of $\mathrm{H}$ II regions and starbursts.

Recent work by Trundle et al. (2002) and Urbaneja et al. (2005) have shown that abundance gradients in some spiral galaxies derived from stellar and nebular studies tend to be coherent but very dependent on the calibration used in the strong line nebular methods. However, until now, there have been no detailed systematic studies that compare results from nebular and stellar studies. This is the first in a series of papers aimed at this subject. We selected some galactic H II regions for a detailed study of the interaction between massive stars and the surrounding ISM, looking for consistency in the derived 


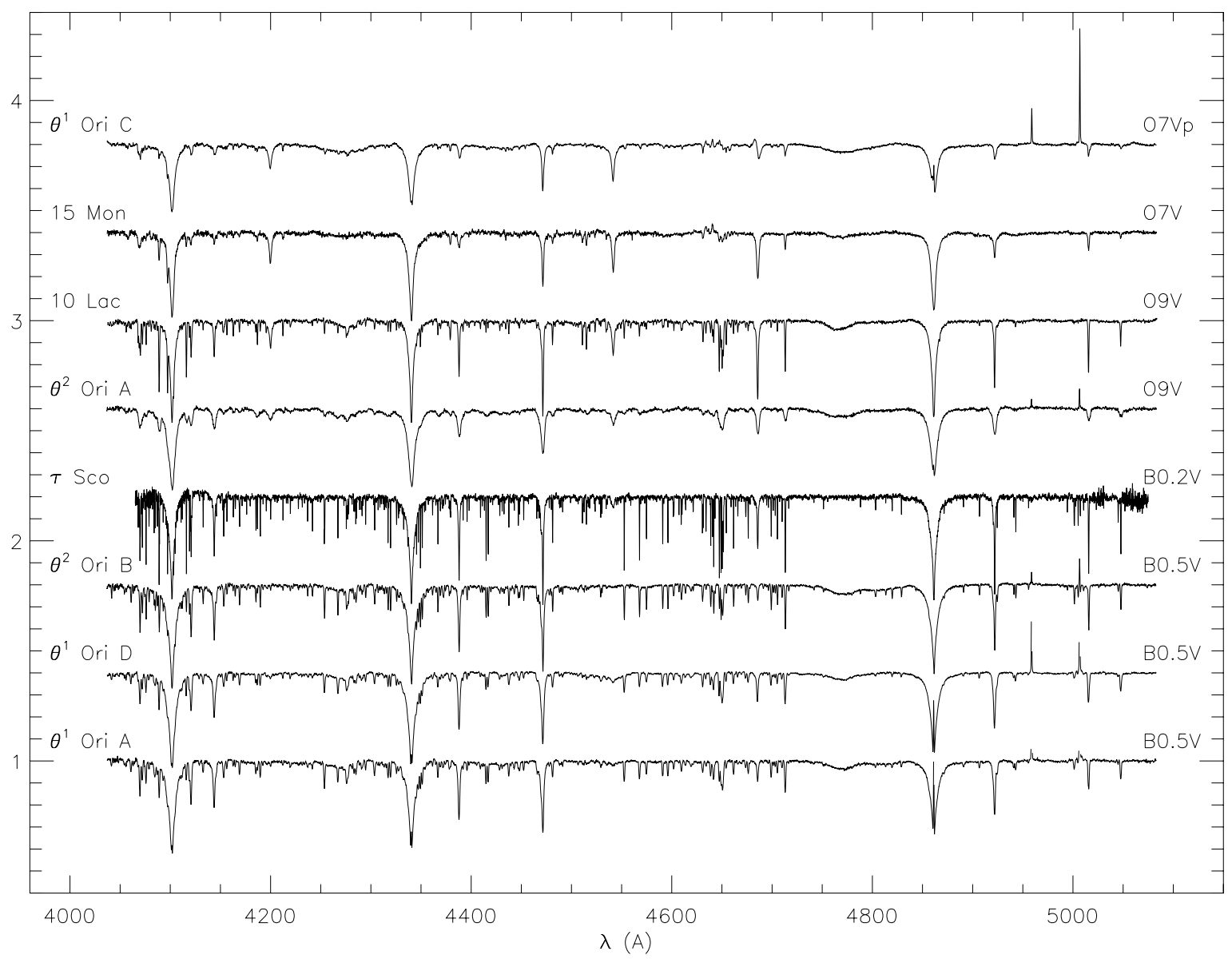

Fig. 1. Atlas of the INT+IDS spectra in the 4000-5000 A region used for this study. The $\tau$ Sco spectrum is from CASPEC. Several narrow nebular emission lines appear in the spectra of the Orion stars. There, the cores of the hydrogen Balmer lines are contaminated by the nebular lines. Nebular $\mathrm{H}_{\beta}$ and [O III] $\lambda \lambda 4960,5007$ lines were arbitrarily diminished in the $\theta^{1}$ Ori A and D plotted spectra for the sake of clarity. Many metal lines can be distinguished, together with the broad $\mathrm{H}$ and $\mathrm{He}$ lines in the $\mathrm{O} 9-\mathrm{B} 0.5$ spectra. The high rotational velocity of $\theta^{2}$ Ori A makes the metal lines of this O9V star shallower, broader, and blended. The known inverted P-Cygni He II $\lambda 4686$ profile can be seen in the $\theta^{1}$ Ori $\mathrm{C}$ spectrum.

parameters ( $T_{\text {eff }}$, luminosities, and ionizing flux distribution of the stellar population), as well as the abundances of $\mathrm{C}, \mathrm{N}, \mathrm{O}$, $\mathrm{Si}$, and $\mathrm{Mg}$. For this first study, we selected the Orion nebula, a well-studied and resolved Galactic H II region powered by a cluster of a few massive stars, the Trapezium cluster.

The Orion complex contains the massive on going starforming region closest to Earth at about only $450 \mathrm{pc}$. The Orion nebula, M 42, is part of this complex. It is a wellknown H II region (e.g. O' Dell 2001; Ferland 2001) ionized by the Trapezium cluster stars $\left(\theta^{1}\right.$ Ori), a group of early type stars located in the core of the nebula. Together with $\theta^{1}$ Ori C (HD 37022, O7V), the main ionizing source, we find other B0.5V stars that are perfect targets for a stellar abundance study.

The most recent study of the chemical composition of the Orion nebula has been presented by Esteban et al. (2004) who re-analyzed the emission line spectrum of the nebula to determine the physical conditions and abundances of the ionized gas-phase. Cunha \& Lambert $(1992,1994)$ included some of the Trapezium cluster stars in a survey of B-type stars in the Orion OB1 association. They presented a spectroscopic analysis of these stars to determine $\mathrm{C}, \mathrm{N}, \mathrm{O}, \mathrm{Si}$, and Fe stellar abundances.

This paper focuses on a spectroscopic analysis of the Trapezium cluster stars to derive their stellar parameters and oxygen abundances. The stellar parameters obtained for $\theta^{1}$ Ori C will be used in future papers as input for modeling the Orion nebula with photoionization codes. The derived stellar abundances are compared to those obtained by Esteban et al. (2004) through nebular studies.

Our paper is structured as follows: in Sect. 2 we present the observations. In Sects. 3 and 4 we obtain the $v \sin i$ and the stellar parameters of our targets. The study of $\theta^{1}$ Ori $C$ is presented in Sect. 5, and the oxygen abundance analyses in Sect. 6. A discussion of the results and the conclusions of this work are presented in Sect. 7.

\section{The observations}

The bulk of the observations used here were carried out with the Isaac Newton $2.5 \mathrm{~m}$. Telescope (INT) at the Roque de los Muchachos Observatory in La Palma on 21 December 2002. The Intermediate Dispersion Spectrograph 


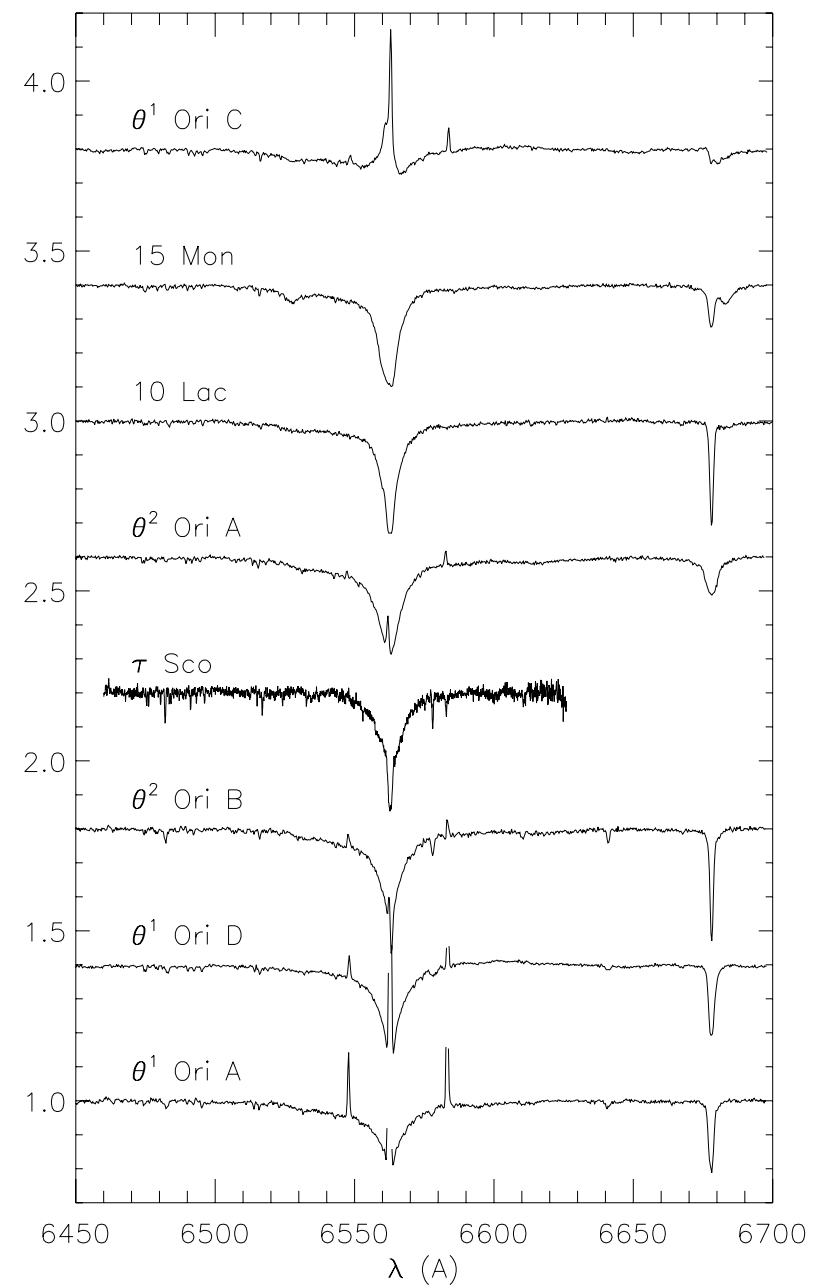

Fig. 2. Atlas of the INT+IDS spectra in the $\mathrm{H}_{\alpha}$ region used for this study. The $\tau$ Sco spectrum is from CASPEC. Nebular $\mathrm{H}_{\alpha}$ and [N II] emission lines have been diminished in the $\theta^{1}$ Ori A and D plotted spectra for the sake of clarity. Two emission components can be distinguished in the $\theta^{1}$ Ori $\mathrm{C}_{\alpha}$ line: the narrow one is associated with the nebula, the other one is variable and it is associated with the star (see Sect. 5). The blue wing of the $\mathrm{H}_{\alpha}$ line is affected by some cosmetic in the INT observations.

(IDS) was used with the $235 \mathrm{~mm}$ camera and two different gratings. We observed the spectral region between 4000 and $5050 \AA$ using the $\mathrm{H} 2400 \mathrm{~B}$ grating, which resulted in an effective spectral resolution $R \sim 7500$ (equivalent to a $0.23 \AA /$ pixel resolution and $\sim 2.6$ pixel $F W H M$ arc lines). The $H 1800 \mathrm{~V}$ grating was used for the $\mathrm{H}_{\alpha}$ region, resulting in a similar spectral resolution ( $0.3 \AA /$ pixel, $R \sim 8000)$. With these configurations, three exposures were needed to cover the whole range. A large number of flat fields and arcs for the reduction process were then obtained.

The reduction and normalization of the spectra was made following standard techniques, with IRAF, and with our own software developed in IDL. The signal-to-noise ratio $(S N R)$ of the reduced spectra depends on the spectral range, but is usually about 200-250 in the blue region and 250 in the $\mathrm{H}_{\alpha}$ region (see Table 2).
Table 1. Identification, spectral type, and photometric visual data of the studied objects. The $A_{\mathrm{v}}$ and $m_{\mathrm{v}}$ values for Orion stars are from Preibisch et al. (1999). The $M_{\mathrm{v}}$ values for these stars were calculated considering a distance $d \sim 450 \pm 50 \mathrm{pc}$ to the Orion nebula. Data for HD 214680 and HD 47839 are from Herrero et al. (1992). Photometric data for HD 149438 are from Humphreys (1978). Uncertainties in $m_{\mathrm{v}}$, $A_{\mathrm{v}}$, and $M_{\mathrm{v}}$ are $0.01,0.03$, and 0.3 , respectively.

\begin{tabular}{cccccc}
\hline \hline HD & Name & SpT & $m_{\mathrm{v}}$ & $A_{\mathrm{v}}$ & $M_{\mathrm{v}}$ \\
\hline Orion stars & & & & & \\
\hline HD 37020 & $\theta^{1}$ Ori A & B0.5V & 6.73 & 1.89 & -3.4 \\
HD 37022 & $\theta^{1}$ Ori C & O7Vp & 5.12 & 1.74 & -4.9 \\
HD 37023 & $\theta^{1}$ Ori D & B0.5V & 6.71 & 1.79 & -3.3 \\
HD 37041 & $\theta^{2}$ Ori A & O9V & 5.07 & 1.12 & -4.3 \\
HD 37042 & $\theta^{2}$ Ori B & B0.5V & 6.41 & 0.73 & -2.6 \\
\hline Reference stars & & & & & \\
\hline HD 47839 & 15 Mon & O7V & - & - & -4.8 \\
HD 214680 & 10 Lac & O9V & - & - & -4.4 \\
HD 149438 & $\tau$ Sco & B0.2V & - & - & -3.3 \\
\hline
\end{tabular}

Table 2. SNR achieved for the different spectra for the three ranges observed with the INT+IDS.

\begin{tabular}{cccc}
\hline \hline HD & $4000-5000$ & $4600-5100$ & $\mathrm{H}_{\alpha}$ \\
\hline HD 37020 & $170-280$ & 210 & 220 \\
HD 37022 & $170-330$ & 280 & 290 \\
HD 37023 & $220-450$ & 450 & 320 \\
HD 37041 & $200-275$ & 230 & 210 \\
HD 37042 & $160-220$ & 250 & 230 \\
HD 214680 & $140-190$ & 200 & 160 \\
HD 47839 & $89-120$ & 160 & 260 \\
\hline
\end{tabular}

We found some problem when rectifying the INT observations in the $\mathrm{H}_{\alpha}$ region. In the blue wing of the $\mathrm{H}_{\alpha}$ line we observed a feature that is independent of rectification. No known line should be present at this wavelength, so we argue that it must be cosmetic.

Special care has to be taken over the nebular contamination of the stellar spectra. The stars we studied are located inside $\mathrm{H}$ II regions, so the stellar spectra are contaminated by the nebular emission spectrum. It would be desirable to remove this nebular contribution, as it fills the cores of the Balmer $\mathrm{H}$ and He I lines. However, this is not easy even though we have long slit observations; the nebular emission has a spatial structure that complicates its subtraction, especially for the Balmer $\mathrm{H}$ lines, the most important nebular contribution. If this is not correctly done, then an over or under subtraction will appear. After trying different possibilities, we concluded that the best solution for this problem is not to subtract the emission lines and to ignore these regions in the final spectrum. For the Balmer $\mathrm{H}$ and $\mathrm{He} \mathrm{I}$ lines, this is satisfactory, as emission lines are narrower than absorption lines. Nebular contributions could be more difficult to separate for metal lines; however, the contamination of the stellar metal lines used for the abundance analysis due to nebular lines is negligible. 
The INT observations consist of the brightest three stars in the Trapezium cluster $\left(\theta^{1}\right.$ Ori A, C, D), together with the two nearby stars $\theta^{2}$ Ori A and B. Two standard stars were included in this set, $10 \mathrm{Lac}$ and $15 \mathrm{Mon}(\mathrm{O} 9 \mathrm{~V}$ and O7V, respectively). The other standard star, $\tau$ Sco, was kindly provided by Dr. Gehren. This is a slow rotating B0.2V star that is perfect for a preliminary abundance analysis. The spectrum was obtained with CASPEC, attached to the ESO $3.6 \mathrm{~m}$ telescope. The $S N R$ of this spectrum is $\sim 200-300$ in the blue region and $\sim 150$ in the $\mathrm{H}_{\alpha}$ region.

For the study of the spectral variability of $\theta^{1}$ Ori $C$, we used FEROS spectra, some of them downloaded from the FEROS database and others kindly provided by Dr. Stahl. These observations were carried out with FEROS at the ESO $1.52 \mathrm{~m}$ telescope in La Silla. The instrument is designed for highdispersion spectroscopy with $R \sim 48000$ in the spectral range 3700-9200 ̊. The achieved SNR is 300 at about $4500-5000 \AA$. Different phase observations were used for the variability study (see Table 7), and is presented in Sect. 5.

\section{Determination of rotational velocities}

The analysis of stellar spectra makes use of a number of free parameters like the micro and macroturbulent velocities and the projected rotational velocity, $v \sin i$. The last one has acquired particular importance in recent times because of the mixing that rotation may induce in the interior of massive stars (e.g. Maeder \& Meynet 2000; Villamariz et al. 2002). However, some methods for determining the rotational velocities do not distinguish between rotation and other surface-broadening mechanisms, like macroturbulence.

Conventionally, $v \sin i$ values are based on linewidth measurements of individual features, mainly metal lines that are apparently free of blends. As the principal broadening mechanism of these lines is stellar rotation, it is possible with sufficient resolution to determine $v \sin i$ from the $F W H M$ of the line. Usually metal lines are used; however, in cases of high rotational velocities or high temperatures, metal lines appear blended or are very weak. Therefore, in these cases, the whole He spectrum is used if the $v \sin i$ is high; if $v \sin i$ is not extremely high, only He I lines are used, as these lines are less affected by pressure broadening than are He II lines. However, the $v \sin i$ derived must be tested with some metal lines (if available), as we are not completely sure that rotation broadening will dominate over pressure broadening.

The Fourier method of determining $v \sin i$ is based on how in Fourier space, convolutions transform into products and how, of the rotation, macroturbulence, natural, and instrumental profiles (turbulence and instrumental assumed Gaussian), only the rotation function has zeros in its Fourier transform. These zeros will appear in the total transform function, while Carroll (1933) showed that the position of the zeros are related to the $v \sin i$. Actually, the frequency of the first zero $\left(\sigma_{1}\right)$ is related to the rotational velocity through

$$
\frac{\lambda}{c} v \sin i \sigma_{1}=0.660
$$

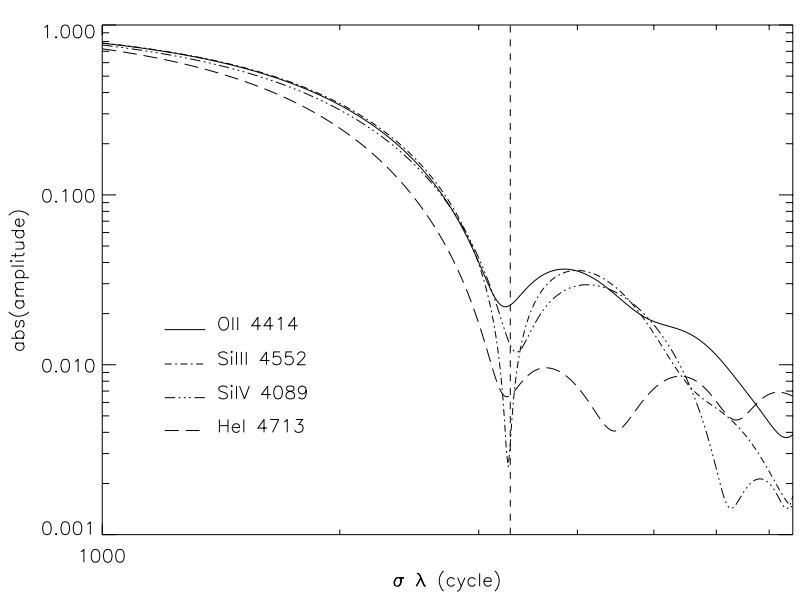

Fig. 3. Four different synthetic lines generated with FASTWIND were convolved with a $v \sin i$ of $60 \mathrm{~km} \mathrm{~s}^{-1}$ and degraded to an $S N R$ of 200. The different lines show the Fourier transform of the lines on a $\sigma \lambda$ baseline. The first zero is very close to the theoretical value for all the lines, even for the case of the He I line.

The microturbulence also introduces zeros in the Fourier transform at high frequencies (Gray 1973). This has to be taken into account for very low $v \sin i\left(\leq 20 \mathrm{~km} \mathrm{~s}^{-1}\right)$.

The main problems in applying of the Fourier method to early type stars are related to the quality of the observed spectra (i.e. spectral resolution and $S N R$ ). The lowest $v \sin i$ limit that can be determined is given by the spectral resolution $(\Delta \lambda$ in $\AA /$ pixel), as the sampling of the computational Fourier transform cannot be extended beyond $0.5 / \Delta \lambda$. The noise in the observed spectra transforms as white noise that obscure the first zero.

The advantages of the Fourier method are that rotational broadening can be separated from other broadening mechanisms and therefore that metal lines, as well as He lines, can be used for the $v \sin i$ determination (even for low values of $v \sin i$ ). This is very useful for fast-rotating stars and spectral types earlier than $\mathrm{O} 9$ that show blended or very weak metal lines.

We tested the Fourier method with theoretical and observational cases, and it works well for massive hot stars. A paper with these results, as well as determinations for a number of O star rotational velocities, is in preparation (Simón-Díaz \& Herrero 2006); Fig. 3 shows a typical example, and for a recent application to A-stars see Royer et al. (2002).

The $v \sin i$ of our sample of stars was determined through the Fourier and FWHM methods. Results are presented in Table 3, together with some values found in the literature for comparison. The resolution in the IDS spectra is $0.23 \AA /$ pixel, so the lowest $v \sin i$ that could be detected with the Fourier method is $\sim 20 \mathrm{~km} \mathrm{~s}^{-1}$. For $\theta^{1}$ Ori C FEROS spectra, the resolution is $\sim 0.03 \AA /$ pixel, so $v \sin i \geq 2 \mathrm{~km} \mathrm{~s}^{-1}$ for detection. For the $\tau$ Sco spectrum, the resolution is $0.1 \AA /$ pixel, so the lowest detectable $v \sin i$ is $8 \mathrm{~km} \mathrm{~s}^{-1}$.

All the papers refered to in Table 3 use the FWHM method applied to the optical spectra of the stars except those by Howarth et al. (1997), who use a cross correlation technique for IUE spectra, and Schönberner et al. (1988), who compare the observed spectrum of $\tau$ Sco with synthetic profiles. 
Table 3. Projected rotational velocity derived from the Fourier and $F W H M$ analyses. References from the literature are: ${ }^{a}$ Simón-Díaz et al. (2003), ${ }^{b}$ Howarth et al. (1997), ${ }^{c}$ McNamara \& Larsson (1962), ${ }^{d}$ Schönberner et al. (1988), ${ }^{e}$ Killian et al. (1991).

\begin{tabular}{cccc}
\hline \hline HD & \multicolumn{3}{c}{$v \sin i\left(\mathrm{~km} \mathrm{~s}^{-1}\right)$} \\
\hline & \multicolumn{2}{c}{ This work } & Other authors \\
\cline { 2 - 4 } & Fourier & $F W H M$ & $F W H M$ \\
\hline HD 37020 & $55.0 \pm 0.6$ & $57 \pm 3$ & $56^{a}$ \\
HD 37022 & $24 \pm 3$ & $40 \pm 11$ & $53^{b}$ \\
HD 37023 & $49.0 \pm 0.9$ & $51 \pm 3$ & $51^{a}$ \\
HD 37041 & $131 \pm 4$ & $140 \pm 11$ & $110^{b}$ \\
HD 37042 & $34.0 \pm 0.5$ & $36 \pm 3$ & $10^{c}$ \\
HD 214680 & $30.0 \pm 0.8$ & $37 \pm 4$ & $35^{b}$ \\
HD 47839 & $67 \pm 4$ & $66 \pm 6$ & $67^{b}$ \\
HD 149438 & $<13$ & $16 \pm 3$ & $5^{d}, 19^{e}$ \\
\hline
\end{tabular}

Comments on the individual stars' $v \sin i$ determination, as well as the comparison between the values derived through Fourier and FWHM methods are presented in Sect. 4.1. Agreement between both methodologies is very good; however, there are some interesting cases (see the study of $\theta^{1}$ Ori C in Sect. 5).

\section{Stellar parameters}

The analyses were performed using the latest version of FASTWIND (an acronym for Fast Analysis of sTellar atmospheres with WINDs), a code originally described by Santolaya-Rey et al. (1997). See Puls et al. (2005) for the newest description of the code along with a discussion of comparisons with previous models and other spherical mass-losing codes. The latest version uses more complete line-blanketing and a temperature correction method based on the energy balance of electrons. The technique used for deriving the stellar parameters is already standard and has been described elsewhere (Herrero et al. 2002; Repolust et al. 2004), so we only give the main points. The analyses are based on visual fitting of hydrogen Balmer lines and He I and He II lines. Through the $\mathrm{He}$ I/He II ionization equilibrium, the effective temperature can be estimated; the wings of the Balmer lines are useful for determining the gravity and can give us some information about the stellar wind.

The code also needs other parameters, such as microturbulence, He abundance, and wind properties (mass loss, terminal velocity and $\beta$ parameter). Actually, wind properties are related through the $Q$ parameter $\left(Q=\dot{M} /\left(v_{\infty} R\right)^{1.5}\right)$.

Once the observed lines are fitted with the modeled ones, effective temperature, gravity, He abundance, microturbulence, and $\log Q$ are determined. The low density in the winds of the studied objects makes the spectrum insensitive to changes in $Q$, so that we can only determine upper values in most cases. Microturbulence has no effect on the $\mathrm{H} / \mathrm{He}$ spectrum of early type stars with large gravity, as has been shown by Villamariz \& Herrero (2002). Therefore only effective temperatures, gravities, and $\mathrm{He}$ abundances were determined for this step of the analysis. Of course, microturbulence is important for the derivation of metallic abundances and will be determined in the corresponding section. The code also provides the emergent flux distribution, so mass, radius, and luminosity can be calculated if $M_{\mathrm{v}}$ is known (see Herrero et al. 1992).

Errors in $T_{\text {eff }}$ and $\log g$ can be established by generating a microgrid around central values; visual comparisons between modeled lines and observations allow us to determine the range of possible values for these parameters. For further comments on the effects of varying the various physical parameters used in the analyses, their mutual interplay and their error bars, see viz. Repolust et al. (2004), Herrero et al. (2002), Villamariz \& Herrero (2002), and Villamariz et al. (2002). Errors in $R, M$ and $L$ are calculated considering the propagations of the uncertainties in $T_{\text {eff }}, \log g$, and $M_{\mathrm{v}}$.

The fits of the synthetic FASTWIND $\mathrm{H}$ and He I-II profiles to the observed ones are shown in Figs. 4 to 10. The derived parameters for our sample of stars are shown in Table 4, corresponding to the best fits. Some comments on the individual analyses and the comparison between spectroscopic and evolutionary results are given in Sects. 4.1 and 4.3.

\subsection{Comments on the Trapezium cluster stars}

HD 37020: this is an eclipsing binary discovered by Lohsen (1975). The period is $P=65.43233 \mathrm{~d}$, the magnitude range $m_{\mathrm{v}}=6.7-7.6$, and the eclipse lasts $\sim 20 \mathrm{~h}$. The primary is a B0.5V star with many isolated metal lines. We did not find lines of the secondary star in our spectrum. This fact, together with the characteristics of the binary system described before, suggest that the secondary is a cooler, smaller, and less luminous star and that the change in the visual magnitude is due to the eclipse of the primary by its companion. Therefore the optical spectrum is mainly dominated by the B0.5V star.

The Fourier method was applied to some O II, Si III-IV, $\mathrm{N}$ II, and He I lines, deriving a $v \sin i=55 \pm 0.6 \mathrm{~km} \mathrm{~s}^{-1}$. Metal and He I lines are in agreement. The $v \sin i$ derived through the linewidth measurement method is consistent with this value (see Table 3 ).

Figure 4 shows the good fit of the FASTWIND profiles to the observed spectra for the parameters given in Table 4, except for the forbidden component of He I $\lambda 4471$ which is not reproduced well throughout our analyses. Note also that the apparently bad fit of He II $\lambda 4200$ is due to the blend with the N III line at the same wavelength. We are very close to the applicability limit of the He I-II ionization equilibrium for deriving the $T_{\text {eff }}$, as He I $\lambda 4200$ and He I $\lambda 4541$ lines are very faint; however, these lines are still sufficiently sensitive to changes in $T_{\text {eff }}$ and $\log g$ for deriving the stellar parameters accurately. The stellar parameters obtained here very well agree with those obtained by Cunha \& Lambert (1992) using the Strömgren index $c_{0}$ and the wings of $\mathrm{H}_{\gamma}$ from the Kurucz's (1979) LTE model atmospheres, though it is not the case for the other two stars in common with these authors. A comparison of values is given in Table 5 . 
Table 4. Stellar parameters derived from FASTWIND analysis. Only an upper limit for $\log Q$ can be derived for these stars. The microturbulences considered for the $\mathrm{HHe}$ analysis in each star are shown in the corresponding fitting plots. A normal value for the He abundance was considered for all the stars $(\epsilon=0.09)$.

\begin{tabular}{cccccccc}
\hline \hline HD & Name & $\begin{array}{c}T_{\text {eff }} \\
\pm 1000 \mathrm{~K}\end{array}$ & $\begin{array}{c}\log g \\
\pm 0.1 \mathrm{dex}\end{array}$ & $R\left(R_{\odot}\right)$ & $M\left(M_{\odot}\right)$ & $\log \left(L / L_{\odot}\right)$ & $\log Q$ \\
\hline HD 37020 & $\theta^{1}$ Ori A & 30000 & 4.0 & $6.3 \pm 0.9$ & $14 \pm 5$ & $4.45 \pm 0.13$ & $<-13.5$ \\
HD 37022 & $\theta^{1}$ Ori C & 39000 & 4.1 & $9.9 \pm 1.4$ & $45 \pm 16$ & $5.31 \pm 0.13$ & - \\
HD 37023 & $\theta^{1}$ Ori D & 32000 & 4.2 & $5.6 \pm 0.8$ & $18 \pm 6$ & $4.47 \pm 0.13$ & $<-13.5$ \\
HD 37041 & $\theta^{2}$ Ori A & 35000 & 4.1 & $8.2 \pm 1.1$ & $39 \pm 14$ & $4.93 \pm 0.13$ & $<-13.5$ \\
HD 37042 & $\theta^{2}$ Ori B & 29000 & 4.1 & $4.5 \pm 0.6$ & $9 \pm 3$ & $4.11 \pm 0.13$ & $<-13.5$ \\
HD 214680 & 10 Lac & 36000 & 3.9 & $8.3 \pm 1.1$ & $20 \pm 7$ & $5.02 \pm 0.13$ & $<-13.5$ \\
HD 47839 & 15 Mon & 40000 & 4.1 & $9.3 \pm 1.3$ & $40 \pm 14$ & $5.30 \pm 0.13$ & -13.0 \\
HD 149438 & $\tau$ Sco & 32000 & 4.0 & $5.6 \pm 0.8$ & $11 \pm 4$ & $4.47 \pm 0.13$ & -13.0 \\
\hline
\end{tabular}

Table 5. Comparison of stellar parameters for HD 37020, HD 37023, HD 37042. The first values refer to the Cunha \& Lambert (1992) determinations, the second values to this work. We see that there is excellent agreement for HD 37020, but poor agreement (specially for $\log g$ ) for the other two stars.

\begin{tabular}{ccc}
\hline \hline Star & $T_{\text {eff }}(\mathrm{K})$ & $\log g($ dex $)$ \\
\hline HD 37020 & $29970 / 30000$ & $3.92 / 4.0$ \\
HD 37023 & $32600 / 32000$ & $4.70 / 4.2$ \\
HD 37042 & $31600 / 29000$ & $4.70 / 4.2$ \\
\hline
\end{tabular}

HD 37022: this is the main ionizing source of the Orion nebula. See Sect. 5 for a detailed study of this star.

$H D$ 37023: of the four Trapezium cluster stars $\left(\theta^{1}\right.$ Ori) this is the only one without a binary companion (Preibisch et al. 1999). Robberto et al. (2004) find indications that this star is surrounded by a photoevaporated circumstellar disk. The Fourier method gives a $v \sin i=49.0 \pm 0.9 \mathrm{~km} \mathrm{~s}^{-1}$ for this star. Again, there is agreement with the linewidth measurement method.

Figure 5 shows the fitting of the HHe lines. Observed He I lines are slightly broader than the theoretical ones. Table 5 compares the stellar parameters we derived with those by Cunha \& Lambert (1992). In this case the agreement is not as good as for HD 37020, although the $T_{\text {eff }}$ are compatible, the $\log g$ they derived is very high.

HD 37041: this is a single-lined spectroscopic binary (see Howarth et al. 1997). These authors find a single peak in the cross correlation function of the IUE spectrum, indicating that the spectrum of the primary is not seriously contaminated.

Comparing the spectrum of this O9V star with that of the standard star $10 \mathrm{Lac}$ (also classified as O9V), we found that there are no unblended metal lines due to its high rotational velocity (except Si IV $\lambda 4089$, but it is in the blue wing of $\mathrm{H}_{\delta}$ ). A good $v \sin i$ determination was possible using the Fourier method with the He I lines. A $v \sin i=131 \pm 4 \mathrm{~km} \mathrm{~s}^{-1}$ was derived. We used the Si IV line to check the reliability of this value; a $v \sin i=136 \pm$ $5 \mathrm{~km} \mathrm{~s}^{-1}$ was obtained. He II does not give good results.
A $v \sin i=140 \pm 11 \mathrm{~km} \mathrm{~s}^{-1}$ was derived using the $F W H M$ method for the He I $\lambda 5015$ line.

Figure 6 shows the fitting of the synthetic profiles with the observed ones. A $v \sin i=131 \mathrm{~km} \mathrm{~s}^{-1}$ was considered for the $\mathrm{H}-\mathrm{He}$ analysis. The wings of the He I lines cannot be well fitted, which might be explained by the presence of a companion.

HD 37042: we found good agreement between metal and He I lines when using the Fourier method. The $v \sin i$ derived is $34.0 \pm 0.5 \mathrm{~km} \mathrm{~s}^{-1}$. The value we obtained with the $F W H M$ method is $36 \pm 3 \mathrm{~km} \mathrm{~s}^{-1}$, in agreement with the former one.

A very good fit of the observed and synthetic FASTWIND profiles was obtained (see Fig. 7). Again the forbidden component of He I $\lambda 4471$ was too weak. For this star, the He I lines fit better if a microturbulence of $10 \mathrm{~km} \mathrm{~s}^{-1}$ was considered.

This is the third star in common with Cunha \& Lambert (see Table 5); we also find for this star (as for HD 37023) that the stellar parameters derived by these authors are very different from ours (they obtain a very high $\log g$ and a higher $\left.T_{\text {eff }} \sim 3000 \mathrm{~K}\right)$.

\subsection{Comments on the reference stars}

HD 214680: this star has been previously considered as a standard for stellar parameter determination through the $\mathrm{H}-\mathrm{He}$ analysis (see Herrero et al. 2002, and references therein). We reanalyzed the spectrum of the star, obtained from a new observing run covering a larger spectral range (the He I $\lambda \lambda 5015,5048,6678$, and He II $\lambda 6683$ lines can be used for the new analysis). We used a new FASTWIND version (Puls et al. 2005), slightly different from the one used by Herrero et al. The new analysis will be useful for checking the consistency of the latest version of the code in this stellar parameter regime.

The projected rotational velocity of this star was determined accurately by means of the FWHM method $\left(v \sin i=37 \pm 4 \mathrm{~km} \mathrm{~s}^{-1}\right)$. The Fourier method applied to the INT+IDS spectrum gives $v \sin i=30 \pm 0.8 \mathrm{~km} \mathrm{~s}^{-1}$. This larger difference could be due to the fact that the 
$v \sin i$ is close to the computational Fourier transform limit ( $20 \mathrm{~km} \mathrm{~s}^{-1}$ for this spectrum), or because the microturbulence is affecting the determination of the rotational first zero in the Fourier transform (Gray 1973).

The whole set of HHe lines is perfectly fitted with the FASTWIND synthetic profiles (Fig. 8). The parameters derived by Herrero et al. (2002) are $T_{\text {eff }}=35500 \mathrm{~K}, \log g=$ 3.95 , and $\epsilon=0.09$. Our results agree with those values.

HD 149438: this star was selected for comparison in the stellar oxygen abundance analysis of the B0.5V Trapezium cluster stars (see Sect. 6), and it has been studied elsewhere (Martin 2004; Przybilla \& Butler 2004; Kilian 1994; Schönberner et al. 1988; Becker \& Butler 1988; Peters \& Polidan 1985; Kane et al. 1980). Comparing isolated oxygen and silicon lines to a set of rotationally broadened profiles, Kilian et al. (1991) obtained a $v \sin i=19 \mathrm{~km} \mathrm{~s}^{-1}$ for this star. The Fourier method applied to the CASPEC spectrum only allows us to say that the $v \sin i$ is lower than $13 \mathrm{~km} \mathrm{~s}^{-1}$; this is because in this case the effect of the microturbulence over the broadening of the metal lines is comparable to that produced by the rotation, so the first zero could be associated with the microturbulence instead of the $v \sin i$ (Gray 1973). A very good fit to the synthetic FASTWIND profiles was obtained for this star (see Fig. 9). In this case the problem with the forbidden component of the He I $\lambda 4471$ can be seen clearly. Table 11 (Sect. 6.4) summarized the stellar parameters obtained in this and previous work.

HD 47839: this star was selected for comparison with the main ionizing source in Orion $\left(\theta^{1}\right.$ Ori C). Some comments on the analysis of this star and the comparison with $\theta^{1}$ Ori $\mathrm{C}$ are presented in Sect. 5. It was classified as O7V(f) by Walborn (1972). Gies (1993) pointed for the first time that this star is a spectroscopic binary with a period of 25 years. He estimated a mass for the primary of $M=34 M_{\odot}$, and suggested that the secondary has a spectral type $09.5 \mathrm{Vn}$ (with very broad lines).

We used the spectrum of HD 214680 (O9V) convolved with a high $v \sin i\left(\sim 350 \mathrm{~km} \mathrm{~s}^{-1}\right)$ for recognizing those lines in the spectrum of HD 47839 not contaminated by the secondary star; three metal lines were found. Using these lines (Si IV $\lambda \lambda 4212,4654$ and N III $\lambda$ 4379) a $v \sin i=$ $67 \pm 4 \mathrm{~km} \mathrm{~s}^{-1}$ was determined. A similar value was derived by applying the FWHM method to the same lines $(66 \pm$ $6 \mathrm{~km} \mathrm{~s}^{-1}$ ).

The fitting of the $\mathrm{H}$ and $\mathrm{He}$ lines for the stellar parameters shows how the He I are contaminated by the secondary star lines. The spectroscopically derived mass $\left(36 \pm 9 M_{\odot}\right)$ agrees very well with the dynamical mass derived by Gies (1993). Herrero et al. (1992) derived a $T_{\text {eff }}=39500 \mathrm{~K}$, $\log g=3.70$, and $\epsilon=0.07$ for this star. Although we expect to obtain a lower $T_{\text {eff }}$ due to including of line-blanketing effects, the value we obtained is slightly higher because there is also a large difference between the $\log g$ we derived (4.0) and the one by Herrero et al. (3.7). There is also another difference, as we do not need a lower He abundance for fitting the He lines. This could be due to a binarity effect; when a composite spectrum is considered in a binary system, the lines can appear diluted or magnified due to the

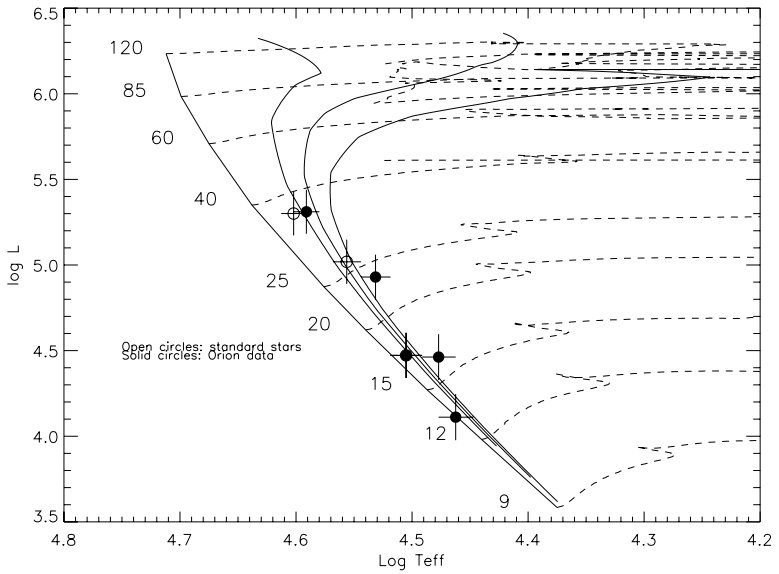

Fig. 11. HR diagram with the studied stars. Evolutionary tracks from Meynet \& Maeder (2003). Isocrones from Schaller et al. (1992), corresponding to 2, 2.5, and $3 \mathrm{Myr}$.

combination of the fluxes of the primary and the companion. If the system is out of eclipse, the total flux will be higher than when the primary is observed isolated, so when the spectrum is normalized all the lines will appear diluted; and then a lower $\mathrm{He}$ abundance is needed to fit the He lines and a lower gravity is derived.

\subsection{Results of the stellar parameters study}

From the optical spectra of the Orion stars, only upper limits for the $Q$ parameter can be achieved. These estimations are based on the effect of the wind on the He II $\lambda 4686$ and $\mathrm{H}_{\alpha}$ lines, with the second contaminated by the nebular contribution. Some tests have shown that the other $\mathrm{H}$ and $\mathrm{He}$ lines are not affected by the uncertainties in $\log Q$ for the range of values considered, so the derived parameters will not be affected.

Masses, radii, and luminosities were derived for all the targets, as indicated in Table 4 together with their uncertainties. The main source of uncertainty for these parameters is the one associated with the absolute magnitude, except for very large uncertainties in $\log g$. An error in $M_{\mathrm{v}} \sim 0.3$ propagates to the mass, radius, and logarithmic luminosity, giving uncertainties of $\sim 37 \%, 13 \%$, and $3 \%$, respectively.

The stars were plotted on the HR diagram in Fig. 11. The evolutionary tracks from Meynet \& Maeder (2003), corresponding to initial masses ranging from 9 to $120 M_{\odot}$ and initial rotational velocities of $0 \mathrm{~km} \mathrm{~s}^{-1}$ were also plotted. All stars are found in the Main Sequence close to the ZAMS, as is expected because of their youth. Nevertheless, we can see the separation from the ZAMS increasing with luminosity, as pointed out by Herrero et al. 2004. The loci of the Orion stars is consistent with an isochrone at about $2.5 \pm 0.5 \mathrm{Myr}$, derived from the tracks with zero initial rotational velocity, which is slightly older than the upper limit given by Palla \& Stahler (1999, 2 Myr) and somewhat larger than other Trapezium age determinations found in the literature (e.g. Hillenbrand 1997, $\leq 1 \mathrm{Myr}$ ). However, it has to be considered that, at large initial rotational velocities, the ZAMS is slightly shifted to the right and modifies the derived ages. Hence, until the role of the 
Table 6. Comparison of masses and gravities derived from the evolutionary tracks and from quantitative analysis of the spectra. The quoted $\log g_{\text {evol }}$ values are given corrected to two decimal places to be consistent with the corresponding evolutionary masses. Note, however, that these are not an indication of the precision of these values, which we consider to be 0.1 dex.

\begin{tabular}{ccccc}
\hline \hline Star & $M_{\text {spec }}\left(M_{\odot}\right)$ & $M_{\text {evol }}\left(M_{\odot}\right)$ & $\log g_{\text {spec }}$ & $\log g_{\text {evol }}$ \\
\hline$\theta^{1}$ Ori A & 14 & 15 & 4.0 & 4.03 \\
$\theta^{1}$ Ori C & 45 & 33 & 4.1 & 3.98 \\
$\theta^{1}$ Ori D & 18 & 16 & 4.2 & 4.16 \\
$\theta^{2}$ Ori A & 39 & 22 & 4.1 & 3.97 \\
$\theta^{2}$ Ori B & 9 & 13 & 4.1 & 4.26 \\
10 Lac & 20 & 25 & 3.9 & 4.02 \\
15 Mon & 40 & 34 & 4.1 & 4.05 \\
$\tau$ Sco & 11 & 16 & 4.0 & 4.16 \\
\hline
\end{tabular}

initial rotational velocities is properly understood (for example, the distribution of initial rotational velocities in clusters), the use of isochones for massive stars in very young clusters should be regarded with special caution.

A good agreement between gravities derived from the evolutionary tracks and those obtained from quantitative analysis of the spectra is found (see Table 6). There is a trend for the most massive stars to have larger spectroscopic than evolutionary masses, but the number of objects is too small to draw any general conclusion.

\section{Modeling the main ionizing star of the Orion nebula}

HD $37022\left(\theta^{1}\right.$ Ori $\left.\mathrm{C}, \mathrm{O} 7 \mathrm{~V}\right)$ is the main ionizing source of the Orion nebula. We want to derive its stellar parameters as a first step in determining the effect of its ionizing flux on the photoionization of the surrounding nebula in a consistent way. Once these parameters are known, the spectral energy distribution can be modeled by using model atmosphere codes. In this way one of the inputs used in the photoionization codes will be fixed consistently.

\subsection{A historical review}

This star is known to have variable spectral features that vary in phase or antiphase with a period of $15.422 \pm 0.002 \mathrm{~d}$ (Stahl et al. 1993; Walborn \& Nichols 1994; Stahl et al. 1996). These variable features were discovered after Conti (1972) showed for the first time that $\theta^{1}$ Ori $\mathrm{C}$ has a variable inverted P-Cygni profile in the He II 4686 line. Among them are $\mathrm{H}_{\alpha}$ emission, variability in the equivalent width of some atmospheric and wind lines, and X-ray emission (Caillault et al. 1994; Gagné et al. 1997). Different explanations for this variability were postulated. The possibility of $\theta^{1}$ Ori $\mathrm{C}$ being a binary and this binarity explaining the spectral variability has been dismissed (Stahl et al. 1996). The variability has been associated with the rotation of the star. Stahl et al. (1996) proposed the presence of a dipolar magnetic field in $\theta^{1}$ Ori $\mathrm{C}$, with the magnetic pole inclined $45^{\circ}$ from the rotation axis (inclined $45^{\circ}$ from the line of sight). The geometry of this system would imply alignment between magnetic pole and the line of sight at phase 0.5 , and they would be perpendicular at phase 0.0 (when maximum $\mathrm{H}_{\alpha}$ emission is found). Babel \& Montmerle (1997) propose the magnetically confined wind-shock model (MCWS) for explaining the variability in the star. According to this model, the radiatively line-driven wind is confined by a dipolar magnetic field towards the magnetic equator of the system, thereby generating both a cold, dense disk due to the collision of material coming from both hemispheres and a hot post-shock region.

The wind characteristics of $\theta^{1}$ Ori $C$ were determined by Howard \& Prinja (1989) and Stahl et al. (1996) through UV spectrum studies. The former derived a mass loss rate of $4 \times 10^{-7} M_{\odot} \mathrm{yr}^{-1}$, the latter determined a terminal velocity somewhat greater than $2500 \mathrm{~km} \mathrm{~s}^{-1}$ through the absorption in $\mathrm{C}$ IV lines. It is common for O7V stars to have stellar winds; what is not so common is the detection of magnetic fields in O stars. Donati et al. (2002) succeeded in detecting Zeeman features in the spectrum of $\theta^{1}$ Ori $C$ through spectropolarimetric observations with the Anglo-Australian Telescope. They detected variability in the Stokes $V$ profiles of some photospheric metal lines. This variability has a coherent modulation with the period derived from other variable features. However, the geometry derived contradictes the one from Stahl et al. (1996), with the magnetic pole aligned with the line of sight at phase 0 , where they found a maximum in the longitudinal component of the magnetic field.

\subsection{Preliminary study of the spectrum}

Once spectral variability is known, it is very important to understand the cause of this variability and to determine which lines are reliable for the stellar atmosphere modeling before comparing synthetic and observed $\mathrm{H}-\mathrm{He}$ profiles. Preliminary work with the INT+IDS spectrum showed that a better spectral resolution was needed to apply the Fourier method to obtain the $v \sin i$. This spectrum allowed us neither to have a good enough sampling $(\Delta \lambda)$ nor to carry on a study of the variability, so we decided to use some FEROS spectra with better quality that covered all variability phases (see Table 7), which are available in the ESO archive.

Through the study of the FEROs spectra observed in the different phases, we have found all the variable spectral features described in Stahl et al. (1996) and some new ones:

- He II 4686: broad emission appears in the blue wing of the line (the so-called inverted P-Cygni profile, with maximum at $\phi \sim 0)$. Broad emission is also present in the red wing (maximum at $\phi \sim 0.5$, minimum at $\phi \sim 0.8$ ).

- Balmer lines: these lines are affected by the same broad emission features as those in He II 24686 . The emission features are stronger in $\mathrm{H}_{\alpha}$ and $\mathrm{H}_{\beta}$ than in the other Balmer lines

- Metal and He I-II lines: all the line strength varies in phase, being larger at $\phi \sim 0$.

This variability can be easily explained considering the model proposed by Stahl et al. (1996) and developed by Babel \& Montmerle (1997). According to this model we would have 


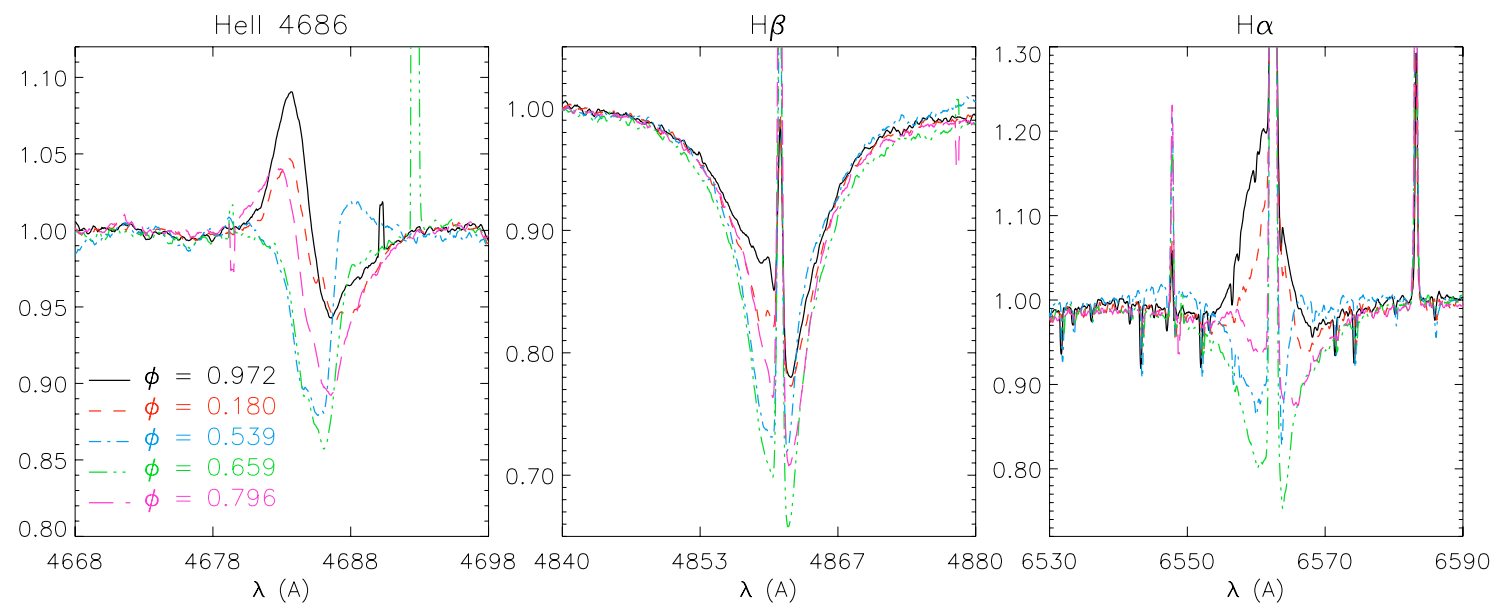

Fig. 12. The most representative phases were selected to show the variability of the He II $\lambda 4686, \mathrm{H}_{\beta}$ and $\mathrm{H}_{\alpha}$ lines in the $\theta^{1}$ Ori $\mathrm{C}$ spectrum. The variable feature associated with the inverted P-Cygni profile discovered by Conti in the He II $\lambda 4686$ line is also present in the hydrogen Balmer lines. Another emission feature can be clearly seen in the red wing of these lines at phase $\sim 0.5$. The narrow emission features in the Balmer lines are nebular lines.

Table 7. FEROS spectra used for the study of the spectral variability of $\theta^{1}$ Ori C. All spectra were downloaded from the ESO-FEROS database except f85221, kindly provided by O. Stahl. The different phases were calculated from $\mathrm{MJD}_{0}-2.400 .000,5=48832,5$ (Stahl et al. 1996), and $P=15.422$ days.

\begin{tabular}{cccc}
\hline \hline Name & Date & MJD-2.400.000,5 & $\phi$ \\
\hline f07341+51 & $16 / 10 / 98$ & 51102.31 & 0.180 \\
f85221 & $26 / 07 / 99$ & 51385.43 & 0.539 \\
f03551+61 & $08 / 10 / 98$ & 51094.28 & 0.659 \\
f04711+21 & $10 / 10 / 98$ & 51096.39 & 0.796 \\
f15241 & $28 / 11 / 98$ & 51145.37 & 0.972 \\
\hline
\end{tabular}

an O7V star with a disc. The disc is produced by the confinement of the wind by a dipolar magnetic field through the magnetic equator. We will have a cool disc with material falling back to the stellar surface. If we consider that at phase 0 the cool disc is seen edge-on, the blue-shifted emission appearing in He II $24686, \mathrm{H}_{\alpha}$ and the other hydrogen Balmer lines can be explained as stellar photons absorbed and reemited with a doppler shift corresponding to the velocity of the disc material falling to the surface of the star (in a process similar to what occurs in a stellar wind but with blue-shifted emission and redshifted absorption). As density in the disc is very high, then a strong blue-shifted emission will appear. At phase 0.5, when the disc is seen face-on, the blue-shifted emission disappears. The emission appearing in the red wings of the former lines could be explained as the effect of the scattering of stellar photons by the wind material confined by the magnetic field and accreting to the disc (see Fig. 12).

The disc will also have continuum emission that will affect the total continuum flux received from the star. This effect will be at its maximum when the disc is face-on because the emitting region is larger at this phase. The variability observed in He I, He II, and metal lines (except for the emission in He I 24686) is only a consequence of this effect. As the total continuum flux is varying with the phase, the normalized spectrum will be affected. All absorption lines will be artificially weaker when the disc continuum emission is at maximum. This variability in the lines can allow us to estimate the amount of continuum flux that comes from the disc, and then the visual magnitude variability. By assuming that at phase 0.0 the lines are not affected by the continuum emission from the disc, we scaled the spectra at the other phases to fit in the former spectrum; the scaling factor will be related to the ratio of visual fluxes (i.e. the difference in magnitudes) between the stellar component and the stellar+disc contribution. This can be seen in Fig. 13. From this study we would expect a variability of $\sim 0.16 \mathrm{mag}$. In their catalogue of suspected variable stars, Kukarkin et al. (1981) found a change in $m_{\mathrm{v}}$ of 0.06 mag (5.10-5.16) for $\theta^{1}$ Ori C. Hipparcos has also classified this star as variable; although Hipparcos data do not show a clear pattern, the median magnitude in Hipparcos system is $4.61 \mathrm{mag}$ and the variability of this magnitude varies between 4.56 and 4.70 , which is agreement with our study.

\subsection{Determining the vsini of $\theta^{1}$ Ori $C$}

It is very important to have a good determination of the projected rotational velocity of this star, as it is supposed that the spectral variability of $\theta^{1}$ Ori $C$ is related to its rotation. The derived $v \sin i$ should be independent of the phase and should be coherent with a period of $\sim 15.4$ days. It is shown in Sect. 5.2 that the profiles of metal, as well as He I and He II lines are dependent on the phase; however, this is only an artificial dependence due to the presence of the disk. Once the spectra of different phases are corrected for the effect of dilution by the disc continuum emission, all the metal, He I and He II lines are independent of the phase, except those related to the disc, see Fig. 12.

The Fourier method allows us to separate pure rotational broadening from other broadening mechanisms that affects the shape of the lines. We used this method with some metal lines at phase $\phi \sim 0$ (see Table 8). A $v \sin i=24 \pm 3 \mathrm{~km} \mathrm{~s}^{-1}$ was 


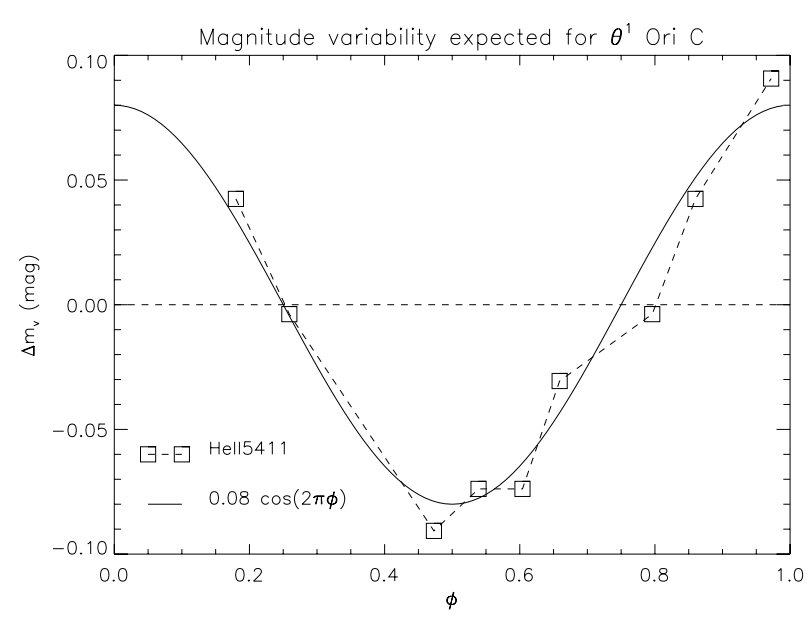

Fig. 13. Magnitude variability expected from the study of the He II $\lambda 5411$ line. The other He I-II lines follow a similar behavior. The presence of a disk could be responsible for this variability (see text for details). The solid line corresponds to a sinusoidal curve with a maximum change in $m_{\mathrm{v}}$ of 0.16 , presented for comparison.

Table 8. Projected rotational velocities derived through Fourier and FWHM methods for some metal lines present in the spectrum of $\theta^{1}$ Ori C. Values were derived at phase 0.972 (see explanation in text).

\begin{tabular}{ccc}
\hline \hline Line & \multicolumn{2}{c}{$v \sin i\left(\mathrm{~km} \mathrm{~s}^{-1}\right)$} \\
& Fourier & $F W H M$ \\
\hline C III 4056 & 23 & $42 \pm 10$ \\
\hline N III 3998 & 23 & $28 \pm 7$ \\
N IV 4057 & 20 & $33 \pm 7$ \\
N IV 5200 & 20 & $32 \pm 10$ \\
\hline Si IV 4089 & 23 & $62 \pm 14$ \\
Si IV 4654 & 30 & $47 \pm 10$ \\
\hline O III 4081 & 21 & $52 \pm 10$ \\
O III 4376 & 26 & $31 \pm 4$ \\
O III 4435 & 25 & $32 \pm 5$ \\
O III 4454 & 27 & $34 \pm 4$ \\
O III 5592 & 25 & $45 \pm 6$ \\
\hline
\end{tabular}

derived. Figure 14 shows an example of the application of the Fourier method in determination of the $v \sin i$ of $\theta^{1}$ Ori C.

Table 7 offers a comparison of the $v \sin i$ values obtained with the Fourier and FWHM methods. We see that the Fourier method gives more consistent values for all these lines. Differences within the $F W H M$ method may reach a factor of 2 and, in fact, we had problems when trying to fit the profile of some of the lines with a Gaussian profile for measuring their linewidth.

The derived value for $v \sin i$ through the Fourier method is also more coherent with an $\mathrm{O} 7 \mathrm{~V}$ star rotating with a 15.422 days period. Considering $R \sim 11 R_{\odot}$, the upper limit for $v \sin i$ is $\sim 35 \mathrm{~km} \mathrm{~s}^{-1}$, so that the inclination of the rotational pole is $i \sim 45$, in agreement with previous results (see next section).

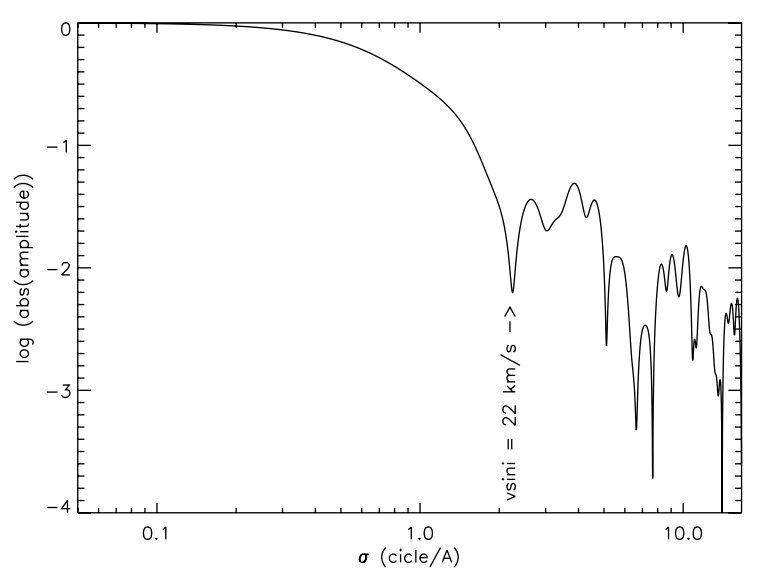

Fig. 14. Fourier analysis of the N IV $\lambda 4057$ line in $\theta^{1}$ Ori C.

\subsection{Modeling of $\theta^{1}$ Ori $C$}

Once the spectral variability of $\theta^{1}$ Ori $\mathrm{C}$ is understood and its $v \sin i$ has been derived, we can proceed to model its stellar atmosphere and wind through the observed spectrum of the star. The lines used for this analysis are shown in Fig. 15; basically, they are the ones used in the other analyses plus He I $5875 \AA$. Some of the lines are contaminated (see Sect. 5.2), so this must be taken into account. The $\mathrm{H}_{\delta}$ and $\mathrm{H}_{\gamma}$ lines were selected as the most reliable lines for deriving $\log g$ (less contaminated than $\mathrm{H}_{\beta}$ and $\mathrm{H}_{\alpha}$ ). The whole set of $\mathrm{He} \mathrm{I}-\mathrm{II}$ lines was considered except He II $\lambda 4686$, but it must be taken into account that the strength of all these lines vary with the phase. Phase 0.972 will be used for determining the stellar parameters, since the effect of the continuum emission of the disc is smaller at this phase (see Sect. 5.2).

Although our study has shown that the rotational velocity (derived from Fourier analysis) is $\sim 24 \mathrm{~km} \mathrm{~s}^{-1}$, when this broadening is considered all synthetic lines appear narrower than the observed ones. We tried to solve the problem by means of a different $v \sin i$ value; however, it does not work, because then the shape of the synthetic profiles do not fit with the observed ones, as the cores of the modeled profiles are too wide when the $F W H M$ of the He lines is fitted. An extra-broadening mechanism has to be included. When a Gaussian macroturbulent broadening (Gray 1973) is used, the fit clearly improves; however, in this case the H I and He II lines cannot be fitted simultaneously with the He I ones, because when the former are fitted (for a $T_{\text {eff }}=39000 \mathrm{~K}$ ), some of the synthetic lines in the latter appear stronger and narrower than observed. A better fitting for the He I lines is obtained if a higher $T_{\text {eff }}$ if considered, but then the synthetic He II lines appear too strong. There is no way to solve this problem in this region of the parameter space either by changing the rotational velocity, the macroturbulence, or the microturbulence.

In Sect. 4.2, Fig. 10 shows that the fitting of the He I-II lines could follow similar behavior in the case of the $07 \mathrm{~V}$ star HD 47839 (selected as reference star). A variation of $1000 \mathrm{~K}$ in the effective temperature strongly changes the strength of the He I lines. For this spectrum it also occurs that when the He I lines appear fitted, the He II lines are slightly stronger than 

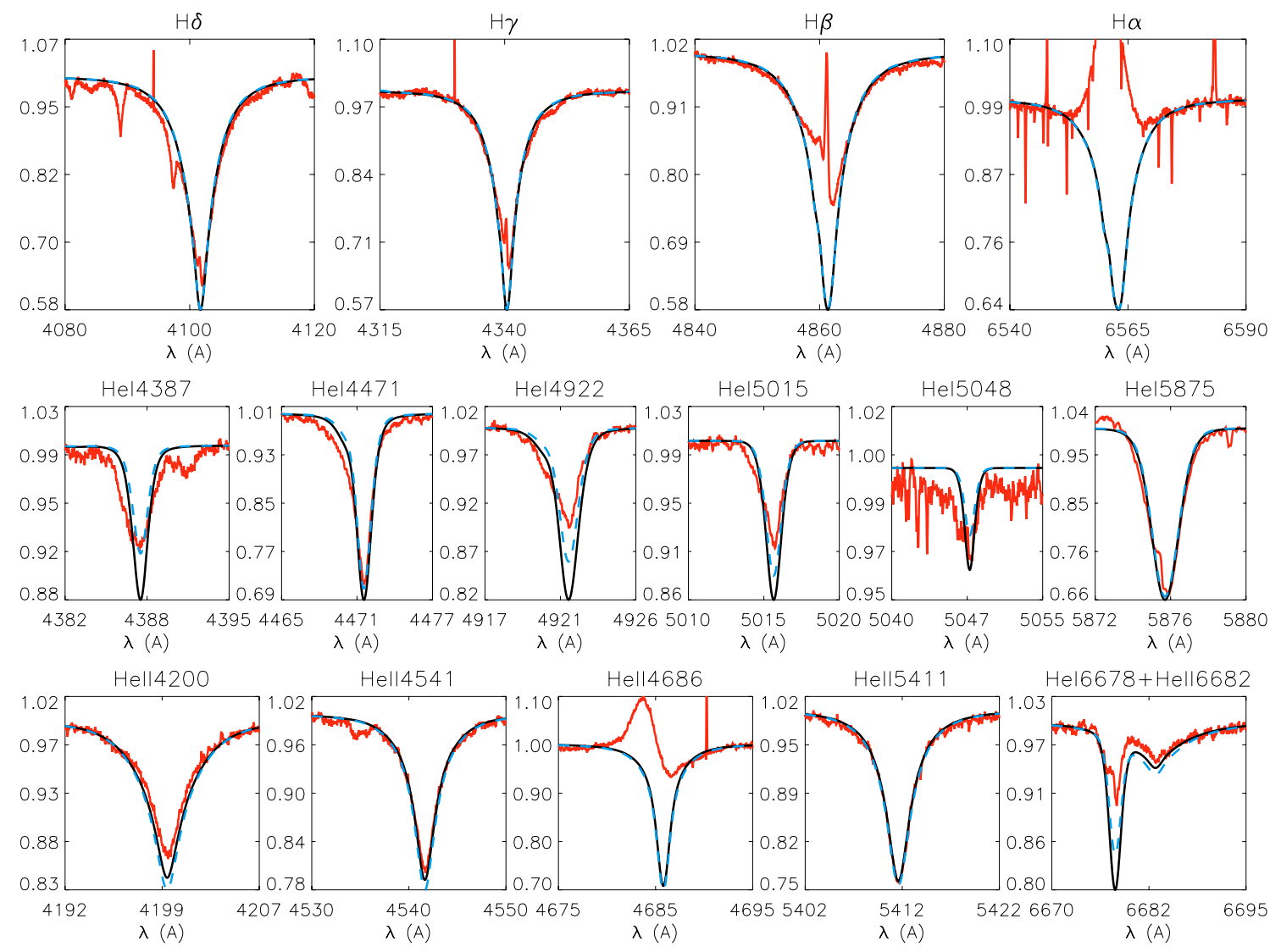

Fig. 15. HHe analysis of HD $37022\left(\theta^{1}\right.$ Ori C, O7Vp) at phase $\phi=0.972$. The solid black line corresponds to a model with $T_{\text {eff }}=39000 \mathrm{~K}$, $\log g=4.1, \epsilon=0.09, \xi_{\mathrm{t}}=5 \mathrm{~km} \mathrm{~s}^{-1}$, and $\log Q=-13.0$; the dashed blue line corresponds to a model with $T_{\text {eff }}=40000 \mathrm{~K}$ (same remaining parameters). A $v \sin i=24 \mathrm{~km} \mathrm{~s}^{-1}$ and a macroturbulent velocity of $60 \mathrm{~km} \mathrm{~s}^{-1}$ were considered. The observed line strength was enlarged by a factor 1.1 to correct for the effect of the disc continuum emission (see text for explanation).

observed, and if the He II lines are fitted, the He I lines are stronger than observed.

Puls et al. (2005) have shown that there is a discrepancy for the HeI singlets between the synthetic FASTWIND and CMFGEN lines for effective temperatures between 36000 and $41000 \mathrm{~K}$ (being the CMFGEN profiles shallower). Therefore the HeI triplet system can be considered more reliable (i.e. the He I $\lambda 4471$ line). Knowing this discrepancy, we considered the He I $\lambda 4471$ for the fitting with FASTWIND synthetic profiles. Our best model corresponds to $T_{\mathrm{eff}}=39000 \pm 1000 \mathrm{~K}$ and $\log g=4.1$ dex. A stellar radius $R=10.6 \pm 1.5 R_{\odot}$ was derived, which implies an inclination of the rotational axis of $i=$ $44 \pm 12^{\circ}$. This value agrees with previous independent studies (Stahl et al. 1996; Donati et al. 2002), although our derived $v \sin i$ is more reliable and our radius is not obtained from an SpT $-R$ calibration, but is the result of the spectral analysis of the star.

\section{Oxygen abundances in the Trapezium Cluster B0.5V stars}

Three of the stars studied in Orion are perfect targets for a stellar abundance analysis, as they have many narrow unblended lines. These are HD 37020, HD 37023, and HD 37042, all three B0.5V stars. Cunha \& Lambert $(1992,1994)$ present carbon, nitrogen, oxygen, and silicon abundances from LTE and
NLTE analyses for these stars. For comparison purposes, a similar analysis has been done for $\tau$ Sco, a B0.2V star (Walborn \& Fitzpatrick 1990) with very low $v \sin i$. Stellar abundances for this star have been derived elsewhere in the literature (Hardorp \& Scholz 1971; Kane et al. 1980; Peters \& Polidan 1985; Schönberner et al. 1988; Becker \& Butler 1988; Kilian et al. 1991; Martin 2004, see Table 11). The other two Orion stars have been ruled out: HD 37041 has a relatively high projected rotational velocity, so metallic lines are broadened and then appear blended; HD 37022, being an O7V star, does not have enough oxygen lines for an accurate abundance analysis.

We therefore derived oxygen abundances for the Trapezium Cluster B0.5V stars for comparing them with the M 42 nebular abundances obtained by Esteban et al. (2004).

\subsection{Line identification and measurement of equivalent widths}

We made use of the curve of growth classical method to determine the oxygen abundances. When this methodology is used, it is important to remove all lines that appear blended. The spectrum of $\tau$ Sco (a star with similar spectral type to our targets in Orion and a very low $v \sin i$ ) was used for an identification of the O II lines present in the spectra of the Orion stars. The whole set of lines is shown in Tables 12 and 13 divided into 
Table 9. Equivalent widths and derived line abundances for the set of O II lines used in our analysis. Line abundances refer to the microturbulence given in brackets for each $\operatorname{star}\left(\xi_{t}\right.$ in $\left.\mathrm{km} \mathrm{s}^{-1}\right)$. Uncertainties in the line abundances come from the propagation of the uncertainties of the equivalent width measurements (see text). Some of the O II lines of the Orion stars have not been used, as they appear blended. O II $\lambda \lambda 4072$, 4076, and 4078 lines were ruled out in the analysis of $\tau$ Sco due to the poor quality of the CASPEC spectrum in this region. Final oxygen abundances for each star were calculated through a weighted mean of the linear values. Errors represent the statistical deviation for these mean values.

\begin{tabular}{|c|c|c|c|c|c|c|c|c|c|c|c|c|}
\hline Line & $E W_{\mathrm{o}}$ & $\epsilon(\mathrm{O})^{a}$ & $\Delta \epsilon(\mathrm{O})$ & $E W_{\mathrm{o}}$ & $\epsilon(\mathrm{O})$ & $\Delta \epsilon(\mathrm{O})$ & $E W_{\mathrm{o}}$ & $\epsilon(\mathrm{O})$ & $\Delta \epsilon(\mathrm{O})$ & $E W_{\mathrm{o}}$ & $\epsilon(\mathrm{O})$ & $\Delta \epsilon(\mathrm{O})$ \\
\hline & \multicolumn{3}{|c|}{ HD $149438\left(\xi_{t}=8.7\right)$} & \multicolumn{3}{|c|}{ HD $37020\left(\xi_{t}=6.5\right)$} & \multicolumn{3}{|c|}{ HD $37023\left(\xi_{t}=7.4\right)$} & \multicolumn{3}{|c|}{ HD $37042\left(\xi_{t}=5.5\right)$} \\
\hline & \multicolumn{2}{|c|}{$T_{\text {eff }}=32000 \mathrm{~K}$} & \multirow{2}{*}{$\begin{array}{l}\log g=4.0 \\
0.04\end{array}$} & \multicolumn{2}{|c|}{$T_{\text {eff }}=30000 \mathrm{~K}$} & $\log g=4.0$ & \multicolumn{2}{|c|}{$T_{\text {eff }}=32000 \mathrm{~K}$} & \multirow{2}{*}{$\frac{\log g=4.2}{-}$} & \multicolumn{2}{|c|}{$T_{\text {eff }}=29000 \mathrm{~K}$} & \multirow{2}{*}{$\frac{\log g=4.2}{-}$} \\
\hline O II 4638 & 85 & 8.75 & & - & - & - & - & - & & - & - & \\
\hline O II 4641 & 127 & 8.65 & 0.04 & - & - & - & - & - & - & - & - & - \\
\hline O II 4661 & 90 & 8.70 & 0.04 & 80 & - & - & 76 & 8.60 & 0.06 & 88 & 8.56 & 0.06 \\
\hline O II 4676 & 79 & 8.71 & 0.05 & 82 & 8.59 & 0.06 & 65 & 8.59 & 0.06 & 82 & 8.63 & 0.06 \\
\hline O II 4317 & 92 & 8.69 & 0.03 & 107 & 8.67 & 0.04 & 78 & 8.59 & 0.05 & 98 & 8.60 & 0.07 \\
\hline O II 4319 & 86 & 8.71 & 0.04 & 100 & 8.69 & 0.05 & 75 & 8.63 & 0.05 & 95 & 8.66 & 0.07 \\
\hline O II 4366 & 80 & 8.61 & 0.04 & 96 & 8.62 & 0.05 & 77 & 8.62 & 0.05 & 103 & 8.62 & 0.08 \\
\hline O II 4416 & 100 & 8.62 & 0.04 & 120 & 8.65 & 0.05 & 93 & 8.62 & 0.05 & 117 & 8.70 & 0.11 \\
\hline O II 4452 & 37 & 8.67 & 0.07 & 48 & 8.64 & 0.07 & 32 & 8.58 & 0.09 & 60 & 8.67 & 0.10 \\
\hline O II 4072 & - & - & - & 118 & 8.61 & 0.12 & 99 & 8.57 & 0.09 & 119 & 8.71 & 0.10 \\
\hline O II 4076 & - & - & - & 133 & 8.59 & 0.12 & 114 & 8.57 & 0.09 & - & - & - \\
\hline O II 4078 & - & - & - & 46 & 8.67 & 0.17 & 33 & 8.59 & 0.09 & 48 & 8.68 & 0.10 \\
\hline O II 4941 & 38 & 8.64 & 0.06 & 50 & 8.68 & 0.07 & 35 & 8.60 & 0.09 & 43 & 8.60 & 0.08 \\
\hline O II 4943 & 60 & 8.62 & 0.04 & 67 & 8.58 & 0.06 & 55 & 8.59 & 0.07 & 56 & 8.48 & 0.07 \\
\hline O II 4956 & 17 & 8.73 & 0.12 & 20 & 8.67 & 0.14 & 13 & 8.58 & 0.20 & 22 & 8.71 & 0.13 \\
\hline O II 4891 & 24 & 8.75 & 0.10 & 27 & 8.68 & 0.11 & 18 & 8.59 & 0.15 & 27 & 8.65 & 0.12 \\
\hline \multirow[t]{2}{*}{ O II 4906} & 34 & 8.64 & 0.07 & 42 & 8.63 & 0.08 & 31 & 8.59 & 0.10 & 47 & 8.72 & 0.08 \\
\hline & \multicolumn{3}{|c|}{$\epsilon(\mathrm{O})=8.70 \pm 0.10$} & \multicolumn{3}{|c|}{$\epsilon(\mathrm{O})=8.65 \pm 0.10$} & \multicolumn{3}{|c|}{$\epsilon(\mathrm{O})=8.59 \pm 0.10$} & \multicolumn{3}{|c|}{$\epsilon(\mathrm{O})=8.64 \pm 0.10$} \\
\hline
\end{tabular}

${ }^{a} \epsilon(\mathrm{O})=\log (\mathrm{O} / \mathrm{H})+12$.

multiplets, together with their $\log g f$ values (basically taken from the NIST database).

The equivalent widths of all the $\mathrm{O}$ II lines listed in Tables 12 and 13 were measured for the four stars; however, only our set of suitable lines (see below) is shown in Table 9. To measure the equivalent widths we use our own software developed in IDL. A least squares profile-fitting procedure was used, with Gaussian profiles fitting the line and polynomials of degree one or two to fit the local continuum. Errors in the measurements due to uncertainty in the position of the local continuum (estimated as $\pm 1 / S N R$, Villamariz et al. 2002) were also considered. The estimated value of the uncertainty in the measurement of the $E W \mathrm{~s}$ is $\sim 5 \mathrm{~m} \AA$ and $\sim 10 \mathrm{~m} \AA$ for some problematic lines.

\subsection{Line selection}

Some of the lines that appear unblended in the spectrum of $\tau$ Sco cannot be used in the analyses of the other stars, since they have larger rotational broadening and then appear blended (or lie in the wings of $\mathrm{H}$ or He lines). Once a first set of unblended lines was selected, a preliminary abundance analysis was done separately for each multiplet. In this way the dispersion in the line abundances for the zero slope value of the microturbulence are minimized as all the lines in a multiplet are formed in the same region in the stellar atmosphere. Problematic lines, errors in the measurement of the equivalent widths or artificial trends can then be identified; such lines will be removed in the global analysis; e.g. this is the case for the O II $\lambda 4414$ line, an isolated line that systematically gives lower abundances.

The set of suitable lines finally used in the abundance analyses is presented in Table 9. They are lines coming from transitions between configurations $2 \mathrm{p}^{2}\left({ }^{3} \mathrm{P}\right) 3 \mathrm{~s}-2 \mathrm{p}^{2}\left({ }^{3} \mathrm{P}\right) 3 \mathrm{p}$ (NIST multiplets 64, 65, and 72) and $2 \mathrm{p}^{2}\left({ }^{3} \mathrm{P}\right) 3 \mathrm{p}-2 \mathrm{p}^{2}\left({ }^{3} \mathrm{P}\right) 3 \mathrm{~d}$ (NIST multiplets 90, 148, and 130). We ruled out the lines that do not follow the general trend and found that lines from multiplets $99,118,161,188,172$ give systematically lower abundances. This effect can be due to the definition of the oxygen model atom we used, or can be associated with the $\log g f$ values. Some preliminary comparisons with TLUSTY analyses have shown that the difference in the line abundances is also present for the case of $\tau$ Sco.

\subsection{Chemical analysis}

For each star we proceed as follows. A grid of 16 FASTWIND models combining four abundances and four values of microturbulence is calculated. In this way, the curves of growth for each line can be constructed by plotting the theoretical equivalent width for each abundance and microturbulence versus the abundance (see Fig. 16 for the case of the O II $\lambda 4414$ line in HD 37042). 


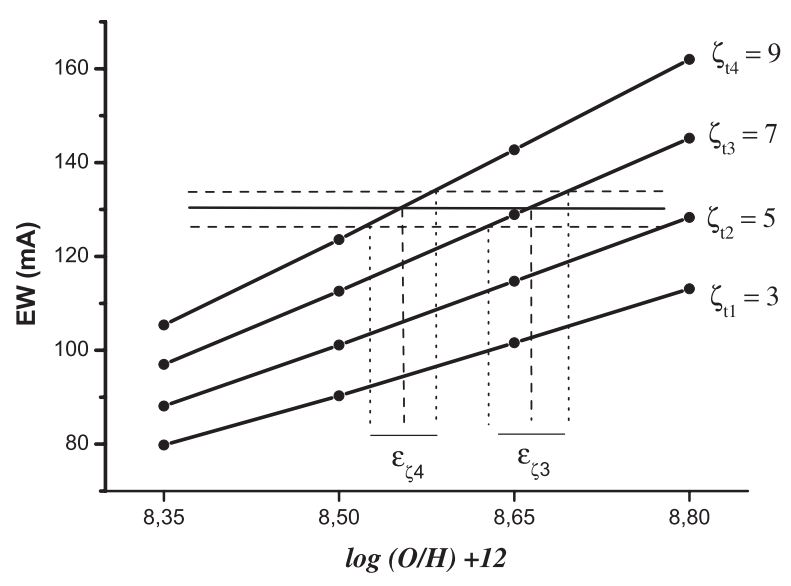

Fig. 16. Example of the curve of growth for the line O II $\lambda 4414$ in the star HD 37042. A grid of 16 models was considered (4 microturbulences and 4 oxygen abundances). The observed $E W$ of the line and its uncertainty are plotted as horizontal lines. Two examples of abundances (and their uncertainties) derived for microturbulences 7 and $9 \mathrm{~km} \mathrm{~s}^{-1}$ are plotted as vertical lines.
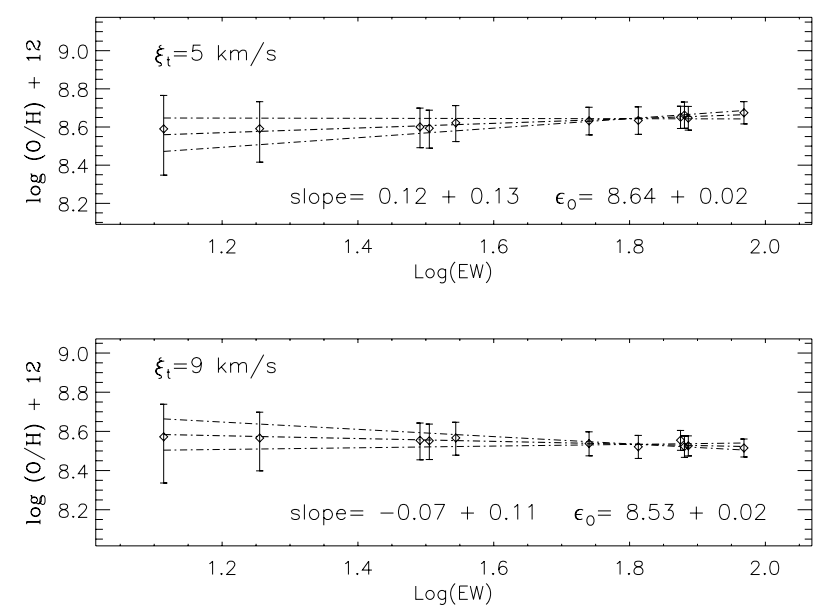

Fig. 17. Example of $\log \epsilon-E W$ diagrams in the study of HD 37023.

Through the observed equivalent width and its error, one abundance (and its derived uncertainty) can be derived for each line and microturbulence. The individual line abundances are dependent on the microturbulence. The microturbulence value that minimizes the dependence of the line abundances on the line strength in the $\log \epsilon-E W$ diagrams will be the microturbulence we are looking for. These diagrams can also be used as a diagnostic tool to check the reliability of the different lines for the abundance determination (see Sect. 6.2). Figure 17 shows the $\log \epsilon-E W$ diagrams for two different microturbulences in the study of HD 37023.

The microturbulence derived from the zero slope for each star is presented in Table 10. Uncertainties in the microturbulence are obtained considering the errors derived for the slope in a linear fit of the data, due to errors in the individual line abundances. This step also allows us to estimate the contribution of the uncertainty in the microturbulence to the total oxygen abundance. This uncertainty depends mainly on the quality of the spectra, in this case $\sim 0.06$ dex.
Table 10. Oxygen abundances for the three B0.5V stars inside Orion nebula and the reference star $\tau$ Sco. Oxygen LTE and NLTE abundances derived by Cunha \& Lambert (1994) for the Orion stars as well as those calculated by Esteban et al. (2004) for the nebula are also presented for comparison.

\begin{tabular}{|c|c|c|c|}
\hline Object & $\mathrm{N}$ & $\xi_{\mathrm{t}}\left(\mathrm{km} \mathrm{s}^{-1}\right)$ & $\log (\mathrm{O} / \mathrm{H})+12$ \\
\hline & & & This work \\
\hline HD 149438 & 14 & $8.1 \pm 1.2$ & $8.70 \pm 0.10$ \\
\hline HD 37020 & 13 & $6.8 \pm 1.3$ & $8.65 \pm 0.10$ \\
\hline HD 37023 & 15 & $6.3 \pm 3.0$ & $8.59 \pm 0.10$ \\
\hline \multirow[t]{2}{*}{ HD 37042} & 13 & $4.9 \pm 2.0$ & $8.64 \pm 0.10$ \\
\hline & & \multicolumn{2}{|c|}{ Cunha \& Lambert (LTE) } \\
\hline HD 37020 & 6 & 7.0 & $8.92 \pm 0.05$ \\
\hline HD 37023 & 7 & 7.5 & $8.76 \pm 0.04$ \\
\hline \multirow[t]{2}{*}{ HD 37042} & 7 & 6.0 & $8.97 \pm 0.04$ \\
\hline & & \multicolumn{2}{|c|}{ Cunha \& Lambert (NLTE) } \\
\hline HD 37020 & 7 & 5.0 & $8.83 \pm 0.12$ \\
\hline HD 37023 & 6 & 7.0 & $8.87 \pm 0.08$ \\
\hline \multirow[t]{2}{*}{ HD 37042} & 6 & 6.0 & $8.85 \pm 0.06$ \\
\hline & & & Esteban et al. \\
\hline Gas & & & $8.65 \pm 0.03$ \\
\hline Gas + dust & & & $8.73 \pm 0.03$ \\
\hline
\end{tabular}

Table 11. Comparison of stellar parameters and abundances derived for $\tau$ Sco in previous studies found in the literature and in this work.

\begin{tabular}{ccccc}
\hline \hline Work & $T_{\text {eff }}(\mathrm{K})$ & $\log g$ & $\epsilon(\mathrm{O})$ & $\xi_{\mathrm{t}}\left(\mathrm{km} \mathrm{s}^{-1}\right)$ \\
\hline Hardorp \& Scholz & 30900 & 4.05 & 8.70 & - \\
Kane et al. & 30300 & 3.95 & 8.6 & - \\
Peters \& Polidan & 31500 & 4.3 & $8.65 \pm 0.26$ & 5 \\
Schönberner et al. & 33000 & 4.15 & 8.5 & 5 \\
Becker \& Butler & 33000 & 4.15 & $8.70 \pm 0.19$ & 5 \\
& & & $8.58 \pm 0.19$ & 10 \\
Kilian et al. & 31400 & 4.24 & $8.61 \pm 0.12$ & 3 \\
Martin & 30000 & 3.75 & $8.58 \pm 0.17$ & 7 \\
This work & 32000 & 4.0 & $8.70 \pm 0.10$ & 8 \\
\hline
\end{tabular}

Abundance values for each line, as well as their uncertainties, are obtained for that microturbulence (see Table 9). The final abundance value is calculated through a weighted mean of the linear individual line abundances $\left(10^{\epsilon_{i}-12}\right)$, and its uncertainty $\left(\sigma_{w}\right)$ is the one associated with this mean. Final values for the total oxygen abundances for each star are shown in Table 10. The final uncertainty in the oxygen abundance takes four different sources of errors into account: those associated with the statistical analysis, those derived from the error in the determined microturbulence and finally those caused by the uncertainty both in the stellar parameters and in the atomic data. All these sources of error are added quadratically to derive the final abundance uncertainty (see Villamariz et al. 2002).

\subsection{Oxygen abundance in the stardard star $\tau$ Sco}

Table 11 summarizes the oxygen abundances appearing in the literature for $\tau$ Sco. Our derived value is compatible with previous results but a little higher than most of them. This 
Table 12. Preliminary set of O II lines selected for the analysis, divided by multiplets. The spectrum of the low $v \sin i \operatorname{star} \tau$ Sco was used to identify the lines. The $\log g f$ values are from the NIST database.

\begin{tabular}{|c|c|c|c|}
\hline Label & $\lambda(\AA)$ & $\log g f$ & Notes \\
\hline \multicolumn{4}{|c|}{$2 s^{2} 2 p^{2}(3 P) 3 p-2 s^{2} 2 p^{2}(3 P) 3 d$} \\
\hline & & \multicolumn{2}{|c|}{ NIST $90\left(4 D_{0}-4 \mathrm{~F}\right)$} \\
\hline \multirow[t]{2}{*}{ O II 4069} & 4069.623 & 0.149 & $+\mathrm{C}_{\text {III }}$ \\
\hline & 4069.882 & 0.344 & \\
\hline O II 4072 & 4072.153 & 0.552 & \\
\hline O II 4076 & 4075.862 & 0.693 & $+\mathrm{C}$ II \\
\hline \multirow[t]{2}{*}{ O II 4078} & 4078.842 & -0.284 & \\
\hline & & \multicolumn{2}{|c|}{ NIST $118\left(2 \mathrm{D}_{0}-2 \mathrm{~F}\right.$} \\
\hline \multirow[t]{3}{*}{ O II 4699} & 4699.218 & 0.269 & \\
\hline & (4699.011 & $0.418)$ & NIST 172 \\
\hline & (4698.437 & $-0.883)$ & NIST 172 \\
\hline O II 4705 & 4705.346 & 0.477 & \\
\hline O II 4741 & 4741.704 & -0.987 & Very weak \\
\hline & & \multicolumn{2}{|c|}{ NIST $130\left(4 \mathrm{~S}_{0}-4 \mathrm{P}\right)$} \\
\hline O II 4891 & 4890.856 & -0.436 & \\
\hline \multirow[t]{2}{*}{ O II 4906} & 4906.830 & -0.160 & \\
\hline & & \multicolumn{2}{|c|}{ NIST $148\left(2 \mathrm{P}_{0}-2 \mathrm{D}\right)$} \\
\hline O II 4941 & 4941.072 & -0.054 & \\
\hline O II 4943 & 4943.005 & 0.239 & \\
\hline O II 4956 & 4955.707 & -0.573 & Very weak \\
\hline \multicolumn{4}{|c|}{$2 s^{2} 2 p^{2}(3 P) 3 s-2 s^{2} 2 p^{2}(3 P) 3 p$} \\
\hline & & \multicolumn{2}{|c|}{ NIST $64\left(4 \mathrm{P}-4 \mathrm{D}_{0}\right)$} \\
\hline O II 4638 & 4638.856 & -0.332 & $+\mathrm{C}$ II $+\mathrm{Si}$ III \\
\hline O II 4641 & 4641.810 & 0.054 & $+\mathrm{N}$ III \\
\hline O II 4650 & 4649.135 & 0.308 & \\
\hline O II 4651 & 4650.638 & -0.362 & \\
\hline O II 4661 & 4661.632 & -0.278 & \\
\hline O II 4673 & 4673.733 & -1.088 & \\
\hline O II 4676 & 4676.235 & -0.394 & \\
\hline \multirow[t]{2}{*}{ O II 4696} & 4696.352 & -1.380 & \\
\hline & & \multicolumn{2}{|c|}{ NIST $65\left(4 \mathrm{P}-4 \mathrm{P}_{0}\right)$} \\
\hline \multirow[t]{2}{*}{ O II 4317} & 4317.139 & -0.386 & \\
\hline & 4317.696 & $\ldots$ & \\
\hline \multirow[t]{2}{*}{ O II 4319} & 4319.630 & -0.380 & $+\mathrm{N}$ III \\
\hline & 4319.866 & -0.502 & \\
\hline \multirow[t]{3}{*}{ O II 4366} & 4366.895 & -0.348 & \\
\hline & 4366.530 & $\ldots$ & \\
\hline & & \multicolumn{2}{|c|}{ NIST $72\left(2 \mathrm{P}-2 \mathrm{D}_{0}\right.$} \\
\hline \multirow[t]{2}{*}{ O II 4414} & 4414.899 & 0.172 & \\
\hline & 4414.456 & -1.483 & \\
\hline O II 4416 & 4416.975 & -0.076 & $+\mathrm{Si} I V$ \\
\hline O II 4452 & 4452.378 & -0.789 & \\
\hline
\end{tabular}

difference can be easily explained by taking into account that, for this range of stellar parameters, the oxygen abundance derived from O II lines is very sensitive to a change in $T_{\text {eff }}$ and $\log g$ : the lines become fainter when a higher $T_{\text {eff }}$ is considered and then the derived oxygen abundance is higher. The uncertainty in the oxygen abundance due to a change of $\sim 1000 \mathrm{~K}$ in $T_{\text {eff }}$ is $\sim 0.08$ dex. This effect is considered in the uncertainty for the given values; however, the central value will
Table 13. (Continued) Preliminary set of O II lines selected for the analysis, divided by multiplets. The spectrum of the low $v \sin i$ star $\tau$ Sco was used to identify the lines. The $\log g f$ values are from the NIST database.

\begin{tabular}{|c|c|c|c|}
\hline Label & $\lambda(\AA)$ & $\log g f$ & Notes \\
\hline \multicolumn{4}{|c|}{$2 s^{2} 2 p^{2}(1 D) 3 s-2 s^{2} 2 p^{2}(1 D) 3 p$} \\
\hline & & \multicolumn{2}{|c|}{ NIST $99\left(2 \mathrm{D}-2 \mathrm{~F}_{0}\right)$} \\
\hline O II 4590 & 4590.974 & 0.350 & \\
\hline \multirow[t]{2}{*}{ O II 4596} & 4595.957 & -1.032 & \\
\hline & 4596.177 & 0.200 & \\
\hline \multicolumn{4}{|c|}{$2 s^{2} 2 p^{2}(1 D) 3 p-2 s^{2} 2 p^{2}$ (1D) $3 d$} \\
\hline & & \multicolumn{2}{|c|}{ NIST $161\left(2 \mathrm{~F}_{0}-2 \mathrm{G}\right)$} \\
\hline O II 4185 & 4185.440 & 0.604 & $+\mathrm{C}$ III \\
\hline \multirow[t]{3}{*}{ O II 4189} & 4189.788 & 0.716 & \\
\hline & 4189.581 & -0.828 & \\
\hline & & \multicolumn{2}{|c|}{ NIST $188\left(2 \mathrm{P}_{0}-2 \mathrm{P}\right)$} \\
\hline \multirow[t]{2}{*}{ O II 4691} & 4690.888 & -0.610 & \\
\hline & 4691.419 & -0.309 & \\
\hline \multirow[t]{4}{*}{ O II 4701} & 4701.179 & 0.088 & \\
\hline & 4701.712 & -0.611 & \\
\hline & 4700.441 & -3.298 & \\
\hline & & \multicolumn{2}{|c|}{ NIST $172\left(2 \mathrm{D}_{0}-2 \mathrm{~F}\right)$} \\
\hline O II 4703 & 4703.161 & 0.263 & \\
\hline
\end{tabular}

slightly depend on the derived stellar parameters. We considered two FASTWIND models with different $T_{\text {eff }}$ (32000, $32500 \mathrm{~K}$ ) and the same $\log g$, thereby obtaining oxygen abundances of 8.70 and 8.74 dex, respectively.

The derived abundance is also very dependent on the chosen microturbulence, especially if lines with high equivalent width are used. We have taken these dependencies into account in our uncertainties.

\subsection{Comparing with nebular and previous stellar results}

The oxygen abundances derived for the Orion stars are compatible within the errors (see Table 10). HD 37023 has a slightly lower abundance, but one still compatible with the other abundances. In Sect. 4.1 we have seen that the fitting of the $\mathrm{H}$ and He lines is not as good as for the other B0.5V stars, since the observed lines appear slightly broader. The oxygen abundances in the Orion stars are systematically lower than that derived for $\tau$ Sco.

Esteban et al. (2004) have recently published a reappraisal of the chemical composition of the Orion nebula. They derived a total oxygen gas-phase abundance $\epsilon(\mathrm{O})=8.65 \pm 0.03$. However, some oxygen is expected to be depleted onto dust grains in ionized nebula, so the total gas+dust oxygen abundance should take this depletion into account. In a previous work (Esteban et al. 1998), these authors estimate the depletion onto dust grains by comparing $\mathrm{Si}$ and Fe nebular abundances with those obtained by Cunha \& Lambert (1994) for B stars in the Orion association, assuming a certain composition for 
the main dust molecules. Taking this correction into account, the final gas+dust oxygen abundance that Esteban et al. (2004) propose is $\epsilon(\mathrm{O})=8.73 \pm 0.03$, where an oxygen abundance correction for dust $\sim 0.08$ is applied.

Our stellar results are compatible with those obtained by Esteban et al. (2004) for the gas phase; however, the dust+gas corrected abundance seems to be too high compared with our derived stellar abundances, although still marginally consistent inside the uncertainties.

Our oxygen abundances are systematically lower than the NLTE abundances by Cunha \& Lambert (1994). In that paper the former authors comment that the LTE abundances are slightly more reliable than the NLTE abundances they present. The difference between their LTE abundances and our results are even higher. These differences can be associated in part with the differences in the derived stellar parameters for these stars. The $T_{\text {eff }}$ and $\log g$ obtained by these authors are higher than ours (see Table 5), so the oxygen abundances they derive are obviously higher.

This difference in the effective temperatures may also affect the derived stellar silicon abundances used by Esteban et al. 1998 for estimating the oxygen depletion. Cunha \& Lambert (1994), derive their silicon abundances by using 3 Si III lines. Preliminary silicon analysis by our group has shown that a difference of $\sim 1000 \mathrm{~K}$ in $T_{\text {eff }}$ can shift the Si III abundances up to 0.2 dex, deriving a higher abundance for the lower $T_{\text {eff }}$.

Alternatively, our result could suggest that the molecules that Esteban et al. use to estimate the $\mathrm{O}$ dust depletion in Orion cannot be present in large amounts in this nebula. Consequently, $\mathrm{Si}, \mathrm{Mg}$, and $\mathrm{Fe}$ (the refractory elements being the main constituents of those molecules) have to form other molecules without oxygen.

\section{Conclusions}

By means of a detailed spectroscopic analysis of the optical spectra of the Trapezium cluster stars, we derived stellar parameters and oxygen abundances. Projected rotational velocities were obtained through Fourier method. This method has been extensively used for late type stars, but not widely applied to early type stars. Our results show this method is very useful for distinguishing between rotational broadening and another broadening mechanisms that can be present in early type stars (e.g. macroturbulence). The agreement is very good when comparing with results from the line-width method. The Fourier method applied to the high resolution $\theta^{1}$ Ori C FEROs spectra allow us to derive a very accurate $v \sin i$ that agrees with the period of variability of some spectral features in $\theta^{1}$ Ori C. Stellar parameters and their uncertainties were derived for these stars using $\mathrm{H}$, He I, and He II lines and the FASTWIND code.

The presence of many $\mathrm{O}$ II lines in the optical spectrum of three B0.5V Orion stars allowed us to work on a very detailed abundance analysis using the curve of growth method. This analysis was performed through a careful selection of suitable lines from a previous study of the different O II multiplets. In this way, the dispersion in the line abundances is reduced, and the final abundance value derived is very precise.
The derived oxygen abundances in the Orion stars agree with the nebular gas-phase abundances obtained by Esteban et al. (2004), and $\sim 0.2$ dex lower than the NLTE abundances derived by Cunha \& Lambert (1994). The gas+dust corrected oxygen abundances estimated by Esteban et al. (1998, 2004), using the Cunha \& Lambert stellar abundances in the Orion association, seem to be too high compared with our derived abundances, although still marginally consistent within the uncertainties. This result suggests a lower dust depletion factor of oxygen than previous estimations for the Orion nebula. A revision of the silicon, magnesium, and iron stellar abundances in the Trapezium cluster stars is presently under way in our group to confirm this result.

Acknowledgements. We want to thank M. A. Urbaneja for the original procedures for deriving the stellar abundances and his invaluable help with FASTWIND, D. Lennon for his comments, and also P. Dufton and R. Ryans for calculating some TLUSTY models for comparison. This work was partially funded by the Spanish Ministerio de Educación y Ciencia under project AYA2004-08271-C02-01. We are very grateful to T. Gehren and O. Stahl for lending us the spectra of $\tau$ Sco and $\theta^{1}$ Ori C. This research made use of the ESO-FEROS database.

\section{References}

Babel, J., \& Montmerle, T. 1997, ApJ, 485, L29

Becker, S. R., \& Butler K. 1988, A\&A, 201, 232

Caillault, J. P., Gagne, M., \& Stauffer, J. R. 1994, ApJ, 432, 386

Carroll, J. A. 1933, MNRAS, 93, 478

Conti, P. S. 1972, ApJ, 174, L79

Crowther, P. A., Hillier, D. J., Evans, C. J., et al. 2002, ApJ, 579, 774

Cunha, K., \& Lambert, D. L. 1992, ApJ, 399, 586

Cunha, K., \& Lambert, D. L. 1994, ApJ, 426, 170

Donati, J. F., Babel, J., Harries, T. J., et al. 2002, MNRAS, 333, 55

Esteban, C., Peimbert, M., Torres-Peimbert, S., et al. 1998, MNRAS, 295, 401

Esteban, C., Peimbert, M., García-Rojas, J., et al. 2004, MNRAS, 355, 229

Ferland, G. F. 2001, PASP, 113, 165

Gagné, M., Caillault, J. P., Stauffer, J. R., et al. 1997, ApJ, 478, L87

Gies, D. R., Mason, B. D., Hartkopf, W. I., et al. 1993, AJ, 106, 2072

Gray, D. F. 1973, ApJ, 184, 461

Hardorp, J., \& Scholz, M. 1970, ApJS, 19, 193

Herrero, A., Kudritzki, R. P., Vilchez, J. M., et al. 1992, A\&A, 261, 209

Herrero, A., Puls, J., \& Najarro, F. 2002, A\&A, 396, 949

Herrero, A., Simón-Díaz, S., Najarro, F., et al. 2004, proceedings of the International Workshop on Massive Stars in interacting binaries (2004, Quebec, Canada)

Hillenbrand, L. A. 1997, AJ, 113, 1733

Hillier, D. J., \& Miller, D. L. 1998, ApJ, 496, 407

Howarth, I. D., \& Prinja, R. K. 1989, ApJS, 69, 527

Howarth, I. D., Siebert, K. W., Hussain, G. A. J., et al. 1997, MNRAS, 284,265

Hubeny, I., \& Lanz, T. 1995, ApJ, 439, 875

Humphreys, R. 1978, ApJSS, 38, 309

Kane, L., McKeith, C. D., \& Dufton, P. L. 1980, A\&A, 84, 115

Kilian, J. 1994, A\&A, 282, 867

Kilian, J., Becker, S. R., Gehren, T., et al. 1991, A\&A, 244, 419

Kukarkin, B. V., Kholopov, P. N., Artiukhina, N. M., et al. 1981, CSV 
Kurucz, R. L. 1979, ApJS, 40, 1

Lohsen, E. 1975, IBVS, 988, 1

Maeder, A., \& Meynet, G. 2000, ARA\&A, 38, 143

Massey, P., Bresolin, F., Kudritzki, R. P., et al. 2004, ApJ, 608, 1001

Massey, P., Puls, J., Pauldrach, A. W. A., et al. 2005, ApJ, 627, 477

Martin, J. C. 2004, AJ, 128, 2474

Martins, F., Schaerer, D., \& Hillier, D. J. 2002, A\&A, 382, 999

Martins, F., Schaerer, D., \& Hillier, D. J. 2005, A\&A, 436, 1049

McNamara, D. H., \& Larsson, H. J. 1962, ApJ, 135, 748

Meynet, G., \& Maeder, A. 2003, A\&A, 404, 975

O’Dell, C. R. 2001, PASP, 113, 290

Palla, F., \& Stahler, S. W. 1999, ApJ, 552, 772

Pauldrach, A. W. A., Hoffmann, T. L., \& Lennon, M. 2001, A\&A, 375, 161

Peters, G. J., \& Polidan, R. S. 1985, IAUS, 111, 417

Preibisch, T., Balega, Y., Hofmann, K. H., et al. 1999, NewA, 4, 531

Przybilla, N., \& Butler, K. 2004, ApJ, 609, 1181

Puls, J., Urbaneja, M. A., Venero, R., et al. 2005, A\&A, in press

Repolust, T., Puls, J., \& Herrero, A. 2004, A\&A, 415, 349

Roberto, M., Beckwith, S. V. W., \& Panagia, N. 2004, AJ, in press
Royer, F., Gerbaldi, M., Faraggiana, et al. 2002, A\&A, 381, 105

Santolaya-Rey, A. E., Puls, J., \& Herrero, A. 1997, A\&A, 323, 488

Schonberner, D., Herrero, A., Becker, S., et al. 1988, A\&A, 197, 209

Simón-Díaz, S., \& Herrero, A. 2006, in preparation

Simón-Díaz, S., Herrero, A., \& Esteban, C. 2003, RMxAC, 18, 123

Smith, M. A., \& Gray, D. F. 1976, PASP, 88, 809

Schaller, G., Schaerer, D., Meynet, G., \& Maeder, A. 1992, A\&AS, 96, 269

Stahl, O., Wolf, B., Gäng, Th., et al. 1993, A\&A, 274, L29

Stahl, O., Kaufer, A., Rivinius, T., et al. 1996, A\&A, 312, 539

Trundle, C., Dufton, P. L., Lennon, D. J., et al. 2002, A\&A, 395, 519

Urbaneja, M. A., Herrero, A., Bresolin, F., et al. 2005, ApJ, in press

Vacca, W. D., Garmany, C. D., \& Shull, J. M. 1996, ApJ, 460, 914

Villamariz, M. R., \& Herrero, A. 2000, A\&A, 357, 597

Villamariz, M. R., Herrero, A., Becker, S. R., et al. 2002, A\&A, 388, 940

Walborn, N. R., \& Fitzpatrick, E. L. 1990, PASP, 102, 379

Walborn, N. R., \& Nichols, J. S. 1994, ApJ, 425, L29

Walborn, N. R. 1972, AJ, 77, 312 


\section{Online Material}


S. Simón-Díaz et al.: Spectroscopic analysis of the Trapezium cluster stars, Online Material p 2
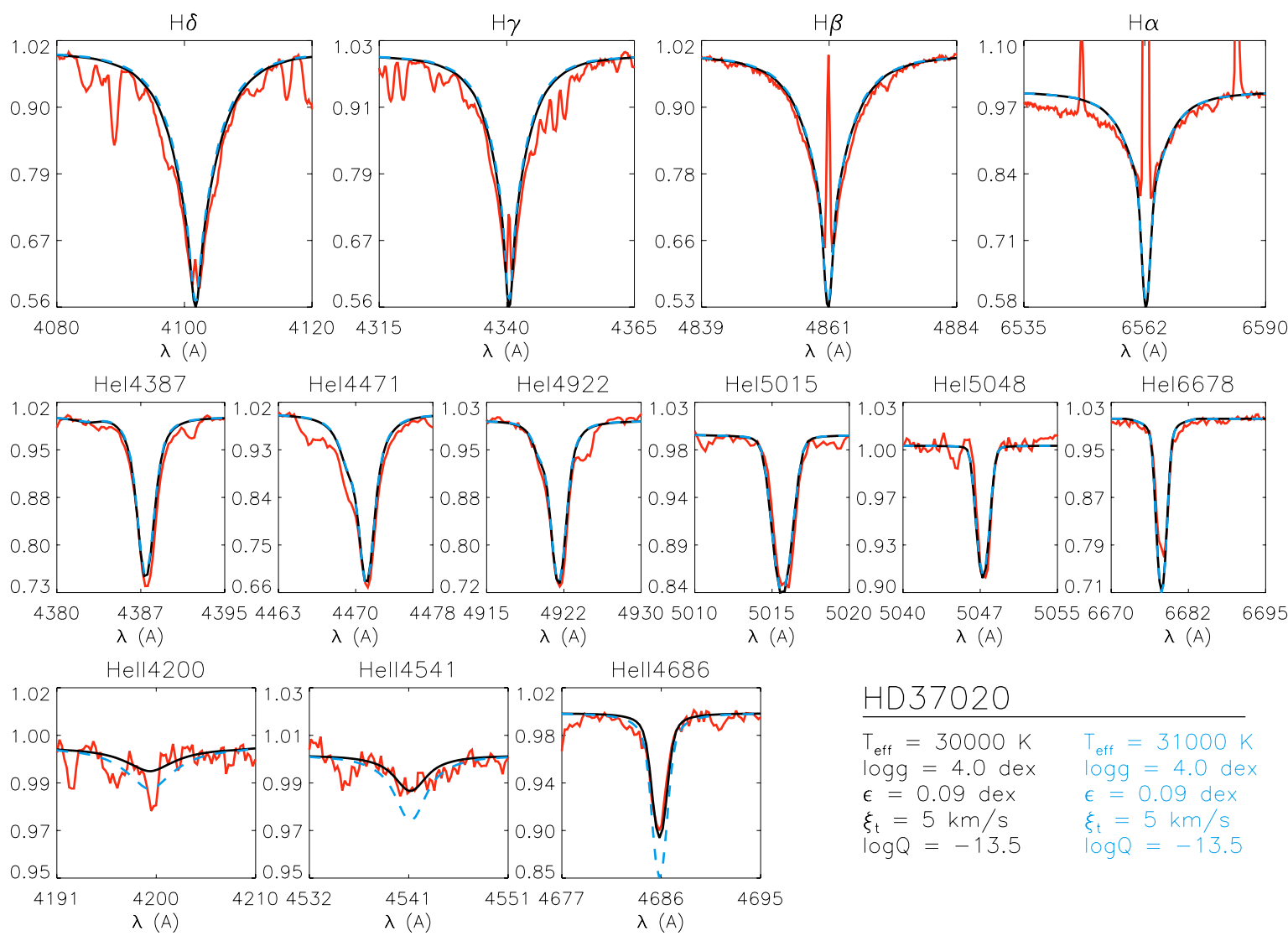

HD37020

\begin{tabular}{ll}
\hline$T_{\text {eff }}=30000 \mathrm{~K}$ & $T_{\text {eff }}=31000 \mathrm{~K}$ \\
$\operatorname{logg}=4.0 \mathrm{dex}$ & $\operatorname{logg}=4.0 \mathrm{dex}$ \\
$\epsilon=0.09 \mathrm{dex}$ & $\epsilon=0.09 \mathrm{dex}$ \\
$\xi_{\mathrm{t}}=5 \mathrm{~km} / \mathrm{s}$ & $\xi_{\mathrm{t}}=5 \mathrm{~km} / \mathrm{s}$ \\
$\log Q=-13.5$ & $\log Q=-13.5$
\end{tabular}

Fig. 4. Analysis of HD $37020\left(\theta^{1}\right.$ Ori A, B0.5V). Two models were plotted for comparison with the observed spectrum. The adopted model is represented with a solid line, while another model varying $1000 \mathrm{~K}$ in $T_{\text {eff }}$ is plotted with a dashed line. Note the core of the stellar Balmer lines contaminated with emission from the nebula. Only the wings of the Balmer lines are used for the fitting. The narrow line that appears in the core of the He II 4200 line is an N III absorption line; the red wing of the He I 4922 line is contaminated by an O II absorption line. The blue wing of the $\mathrm{H}_{\alpha}$ line is affected by a cosmetic feature and is not used for the fitting. 
S. Simón-Díaz et al.: Spectroscopic analysis of the Trapezium cluster stars, Online Material p 3
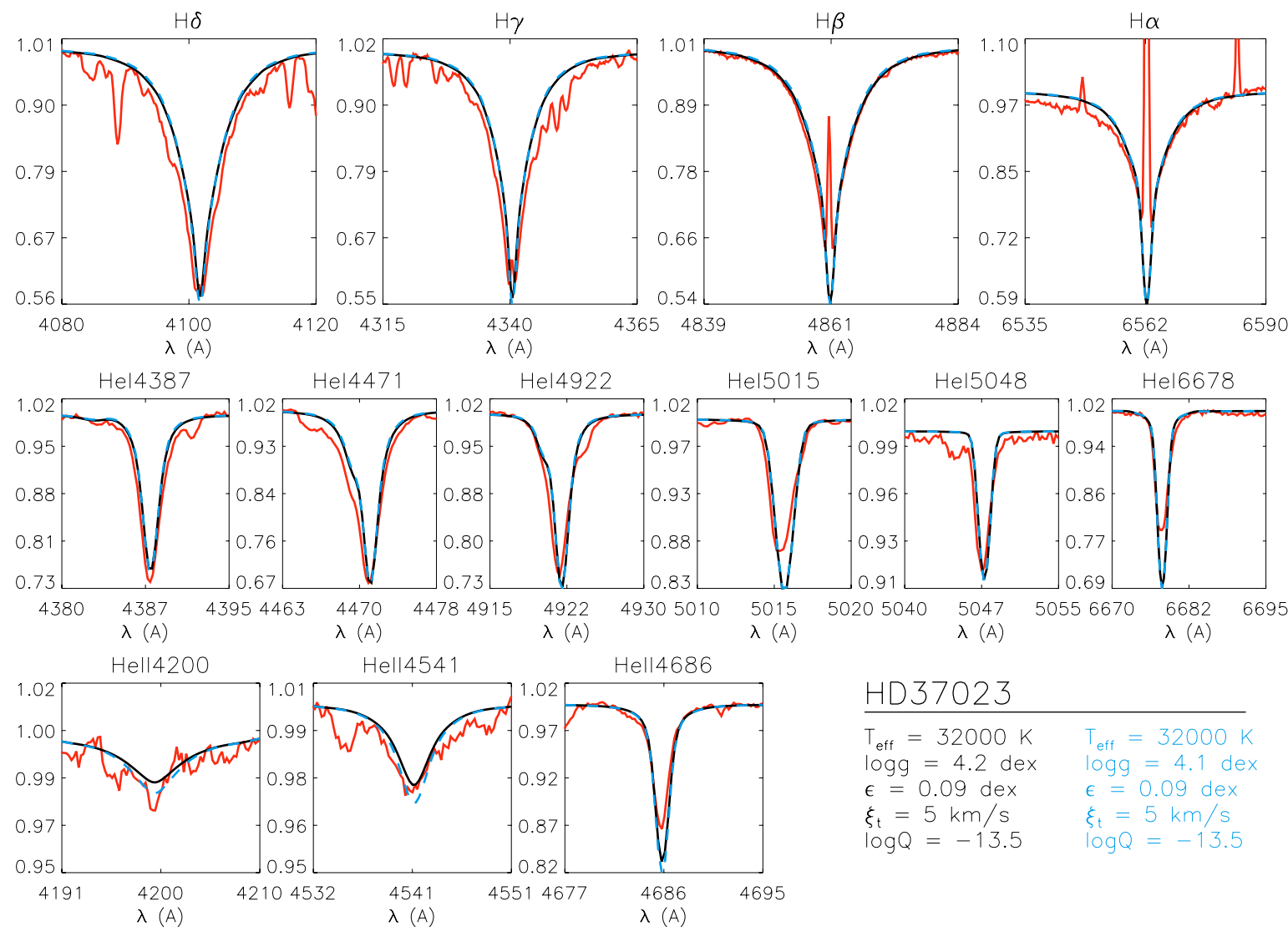

HD37023

$\begin{array}{ll}T_{\text {eff }}=32000 \mathrm{~K} & \mathrm{~T}_{\text {eff }}=32000 \mathrm{~K} \\ \log =4.2 \mathrm{dex} & \log =4.1 \mathrm{dex} \\ \epsilon=0.09 \mathrm{dex} & \epsilon=0.09 \mathrm{dex} \\ \xi_{\mathrm{t}}=5 \mathrm{~km} / \mathrm{s} & \xi_{\mathrm{t}}=5 \mathrm{~km} / \mathrm{s} \\ \log \mathrm{Q}=-13.5 & \log \mathrm{Q}=-13.5\end{array}$

$\log Q=-13.5 \quad \log Q=-13.5$

Fig. 5. As in Fig. 4 for HD $37023\left(\theta^{1}\right.$ Ori D, B0.5V). A variation of 0.1 dex in $\log g$ was considered in this case. 
S. Simón-Díaz et al.: Spectroscopic analysis of the Trapezium cluster stars, Online Material p 4
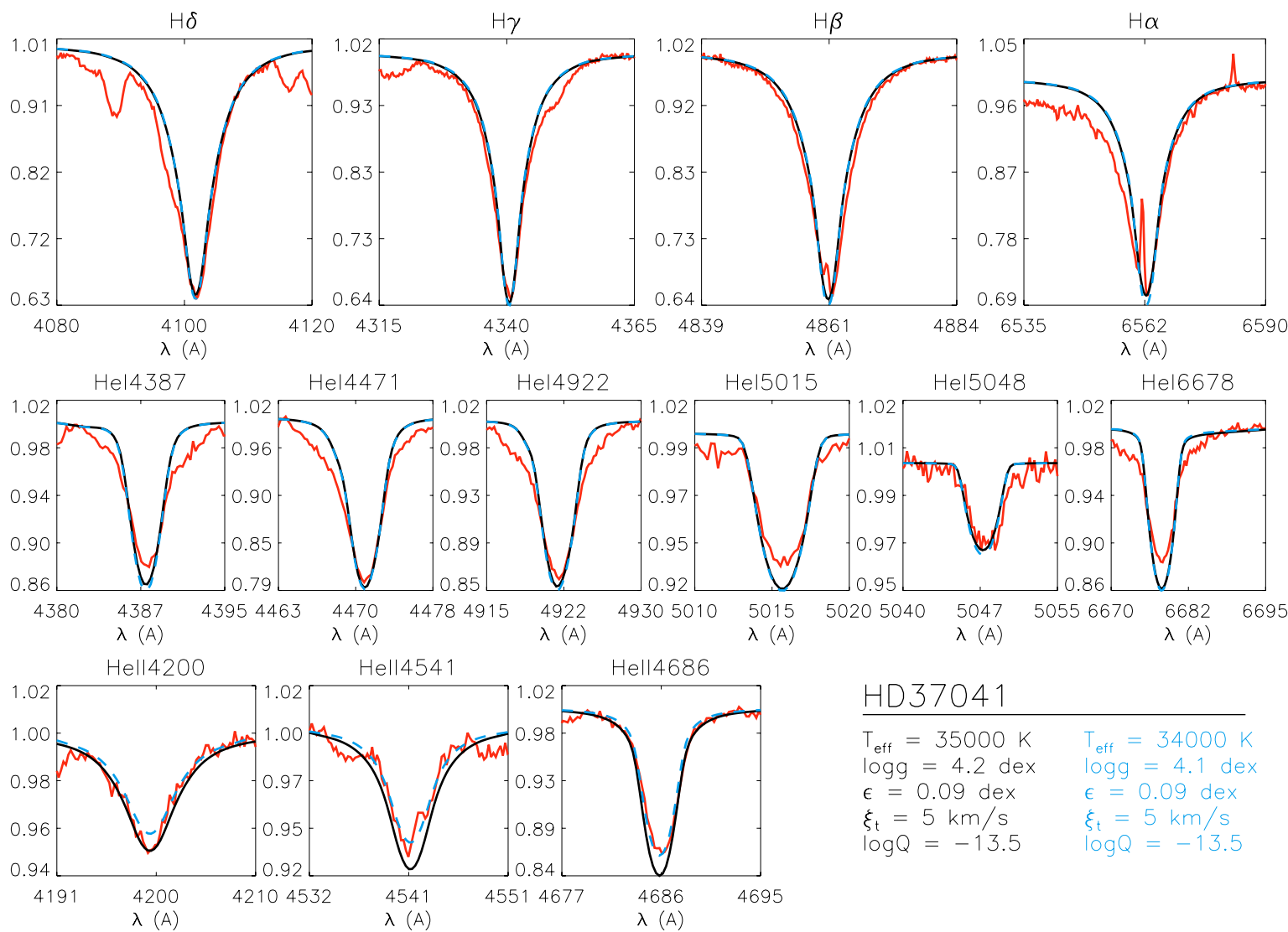

HD37041

$\mathrm{T}_{\text {eff }}=35000 \mathrm{~K} \quad \mathrm{~T}_{\mathrm{eff}}=34000 \mathrm{~K}$ $\log =4.2 \mathrm{dex} \quad \log g=4.1 \mathrm{dex}$ $\epsilon=0.09 \mathrm{dex}$ $\xi_{t}=5 \mathrm{~km} / \mathrm{s}$ $\xi_{\mathrm{t}}=5 \mathrm{~km} / \mathrm{s}$

Fig. 6. As in Fig. 4 for HD $37041\left(\theta^{2}\right.$ Ori A, O9V). A variation of $1000 \mathrm{~K}$ in $T_{\text {eff }}$ along with 0.1 dex in $\log g$ was considered in this case. Note that the broad wings of the He I cannot be fitted correctly (see text). 
S. Simón-Díaz et al.: Spectroscopic analysis of the Trapezium cluster stars, Online Material p 5
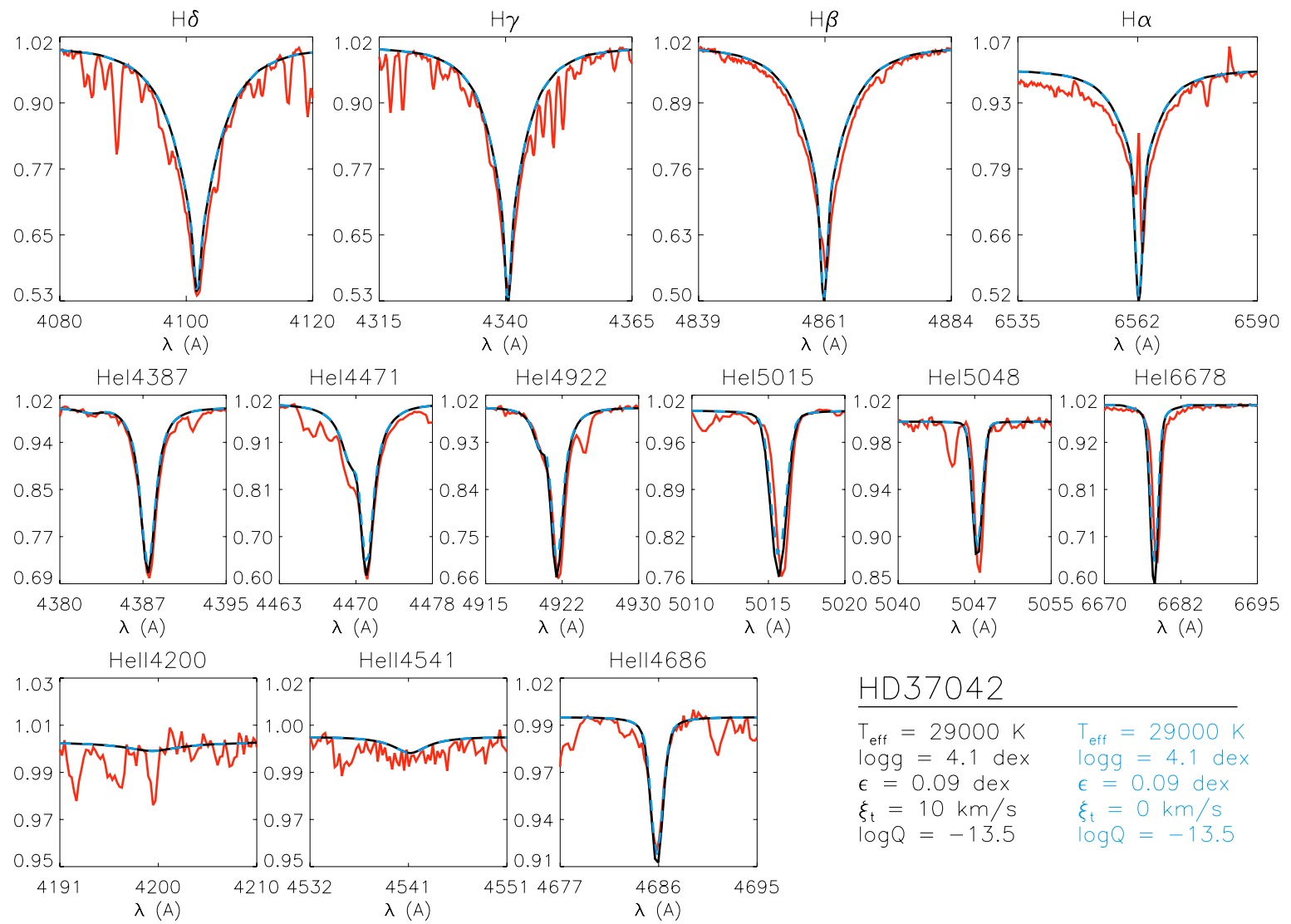

HD37042

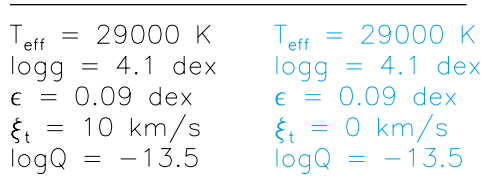

$\log Q=-13.5 \quad \log Q=-13.5$

Fig. 7. As in Fig. 4 for HD 37042 ( $\theta^{2}$ Ori B, B0.5V). For this star, the He I lines fit better if a microturbulence of $10 \mathrm{~km} \mathrm{~s}^{-1}$ is considered. 
S. Simón-Díaz et al.: Spectroscopic analysis of the Trapezium cluster stars, Online Material p 6
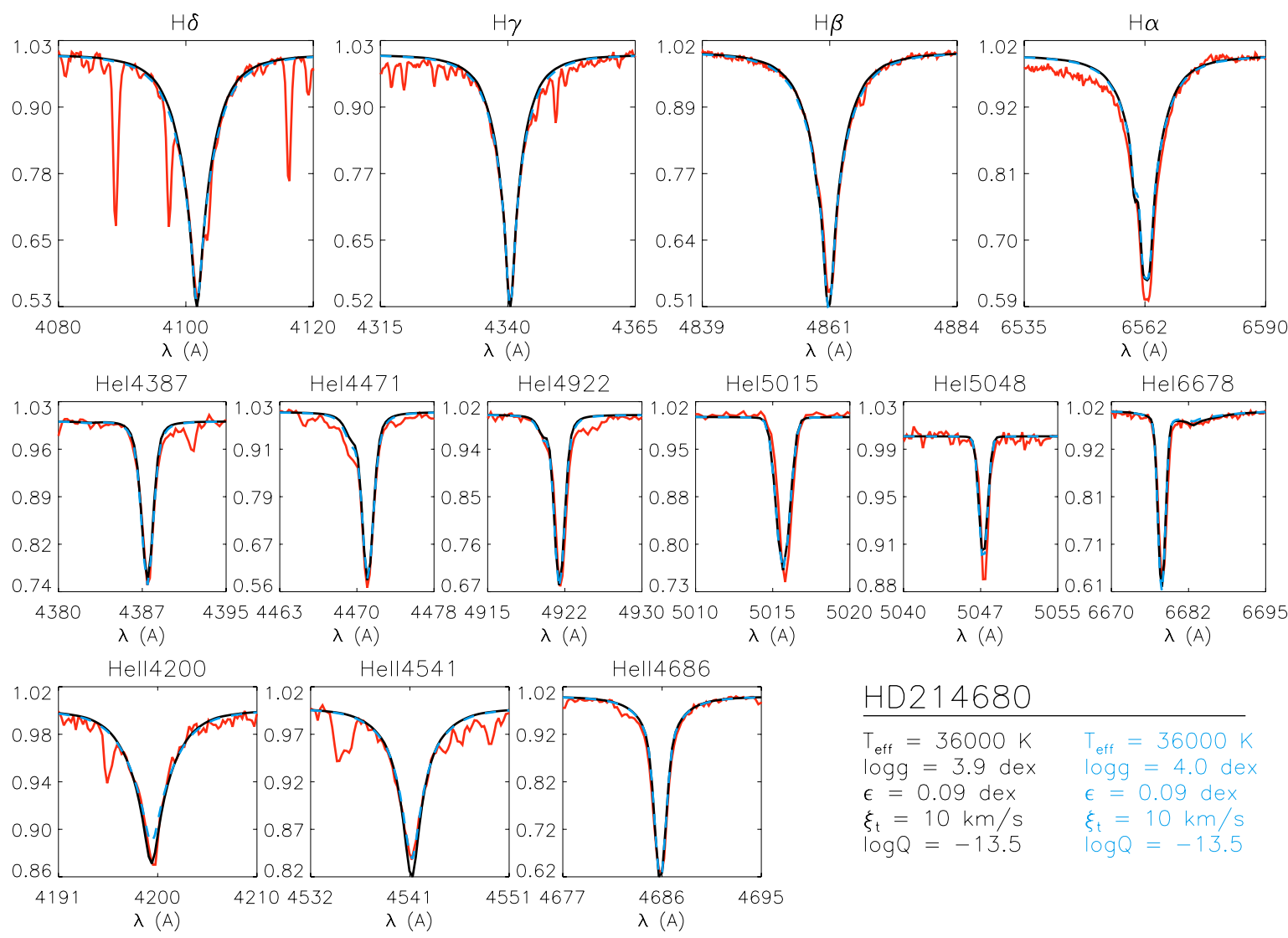

HD214680

$T_{\text {eff }}=36000 \mathrm{~K} \quad T_{\text {eff }}=36000 \mathrm{~K}$ $\log g=3.9$ dex $\quad \log g=4.0$ dex $\epsilon=0.09 \mathrm{dex}$ $\epsilon=0.09 \mathrm{dex}$ $\xi_{\mathrm{t}}=10 \mathrm{~km} / \mathrm{s} \quad \xi_{\mathrm{t}}=10 \mathrm{~km} / \mathrm{s}$ $\log Q=-13.5 \quad \log Q=-13.5$

Fig. 8. As in Fig. 4 for HD 214680 (10 Lac, O9V). A variation of 0.1 dex in $\log g$ was considered in this case. 
S. Simón-Díaz et al.: Spectroscopic analysis of the Trapezium cluster stars, Online Material $p 7$
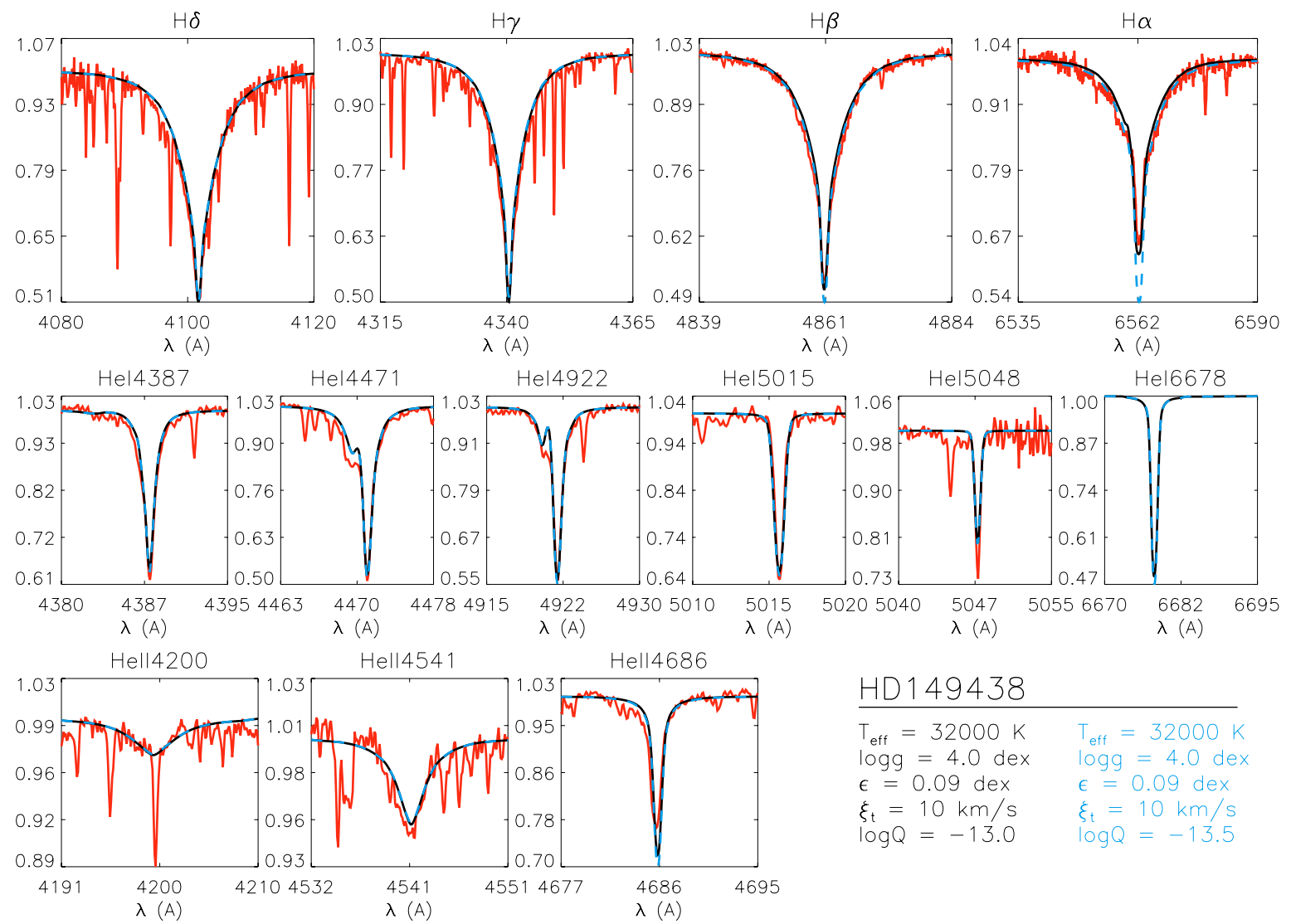

HD 149438

$\mathrm{T}_{\text {eff }}=32000 \mathrm{~K} \quad \mathrm{~T}_{\text {eff }}=32000 \mathrm{~K}$ $\log g=4.0$ dex $\quad \log g=4.0$ dex $\epsilon=0.09 \mathrm{dex}$ $\xi_{\mathrm{t}}=10 \mathrm{~km} / \mathrm{s}$ $\log Q=-13.0$

$\epsilon=0.09 \mathrm{dex}$

$\epsilon=0.09 \mathrm{dex}$
$\xi_{t}=10 \mathrm{~km} / \mathrm{s}$

$\log Q=-13.5$

Fig. 9. As in Fig. 4 for HD 149438 ( $\tau$ Sco, B0.2V). A variation of 0.5 dex in $\log Q$ was considered in this case. The $\mathrm{H}_{\alpha}$ line is not contaminated by nebular emission for this star and a more accurate value of $\log Q$ can be determined. The He I $\lambda 6678$ is out of the observed range in the CASPEC spectrum of $\tau$ Sco. 
S. Simón-Díaz et al.: Spectroscopic analysis of the Trapezium cluster stars, Online Material p 8
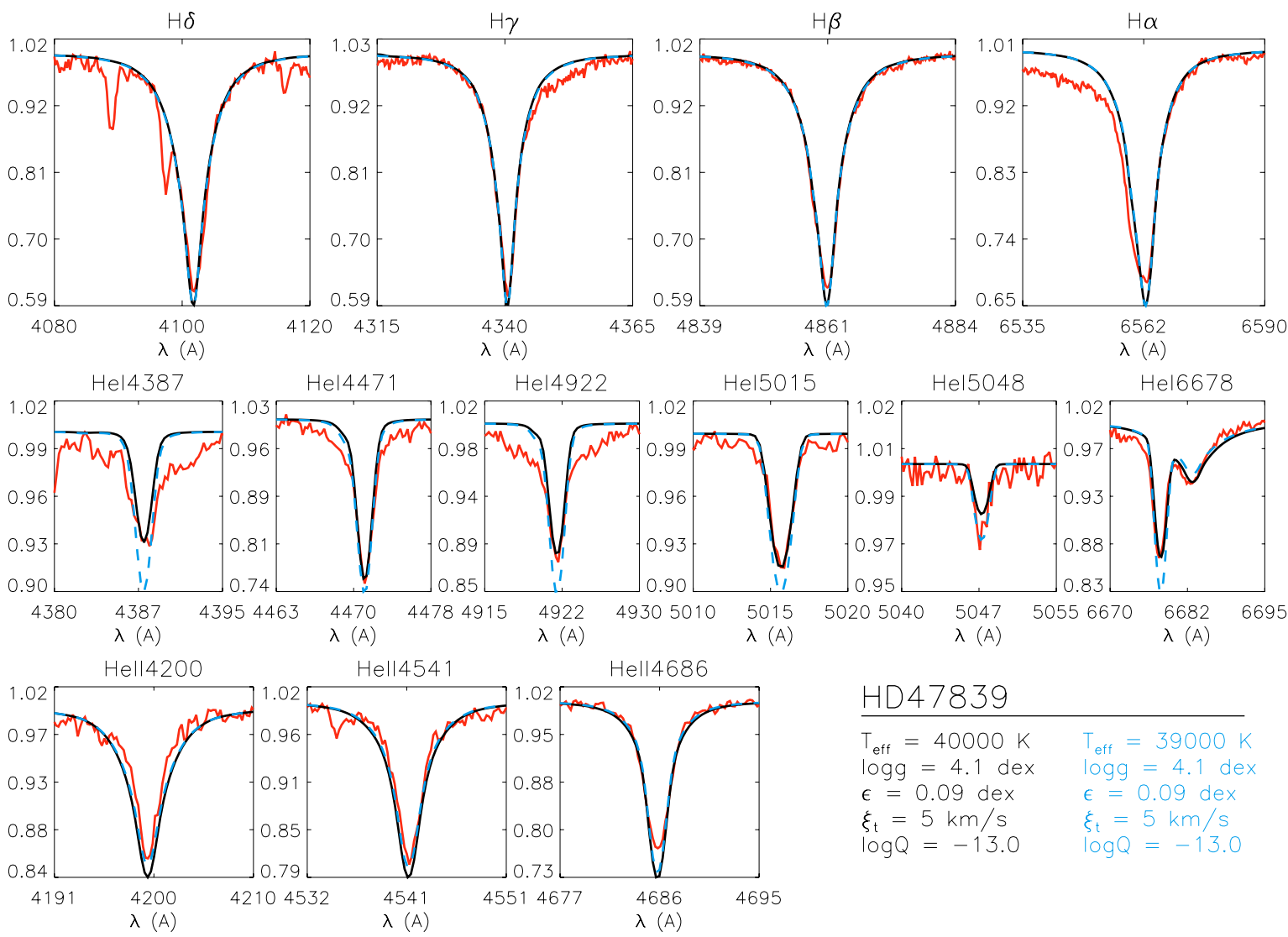

HD47839

$\mathrm{T}_{\text {eff }}=40000 \mathrm{~K} \quad \mathrm{~T}_{\text {eff }}=39000 \mathrm{~K}$ $\log =4.1$ dex $\quad \operatorname{logg}=4.1$ dex $\epsilon=0.09 \mathrm{dex} \quad \epsilon=0.09 \mathrm{dex}$ $\xi_{t}=5 \mathrm{~km} / \mathrm{s} \quad \xi_{t}=5 \mathrm{~km} / \mathrm{s}$ $\log Q=-13.0$

Fig. 10. As in Fig. 4 for HD 47839 (15 Mon, O7V). A variation of $1000 \mathrm{~K}$ in $T_{\text {eff }}$ was considered in this case. 